\title{
Total Synthesis of $( \pm)$-Kalihinol C
}

\author{
Ryan D. White, Gregg F. Keaney, Corin D. Slown, and John L. Wood* \\ Sterling Chemistry Laboratory, Department of Chemistry, \\ Yale University, New Haven, Connecticut 06520-8107
}

\section{Material and Methods}

Unless otherwise stated, reactions were performed under a nitrogen atmosphere using freshly distilled solvents. Diethyl ether $\left(\mathrm{Et}_{2} \mathrm{O}\right)$ and tetrahydrofuran (THF) were distilled from sodium/benzophenone. Methylene chloride $\left(\mathrm{CH}_{2} \mathrm{Cl}_{2}\right)$, and benzene were distilled from calcium hydride. Methanol $(\mathrm{MeOH})$ was distilled from magnesium. All other commercially obtained reagents were used as received. All reactions were magnetically stirred and monitored by thin-layer chromatography (TLC) using E. Merck silica gel 60 F254 pre-coated plates $(0.25-\mathrm{mm})$. Column or flash chromatography was performed with the indicated solvents using silica gel (particle size 0.032-0.063 nm) purchased from Bodman. ${ }^{1} \mathrm{H}$ and ${ }^{13} \mathrm{C}$ NMR spectra were recorded on Bruker Advance DPX-500 or Bruker Advance DPX-400 spectrometers. Chemical shifts are reported relative to internal solvent as described by Gottlieb (i.e. chloroform ${ }^{1} \mathrm{H} \delta 7.26 \mathrm{ppm},{ }^{13} \mathrm{C} \delta$ $77.16 \mathrm{ppm}$; acetone ${ }^{1} \mathrm{H} \delta 2.05 \mathrm{ppm},{ }^{13} \mathrm{C} \delta 29.84 \mathrm{ppm}$; methanol ${ }^{1} \mathrm{H} \delta 3.31 \mathrm{ppm},{ }^{13} \mathrm{C} \delta$ $49.00 \mathrm{ppm})$. Melting points were obtained on a Gallenkamp variable temperature melting point apparatus and are uncorrected. Infrared spectra were recorded on a Midac M-1200 FTIR. High resolution mass spectra were acquired at The University of Illinois Mass Spectrometry Center.

\section{Experimental Procedures}

\section{Preparation of TBS-ethers 6 and 25}

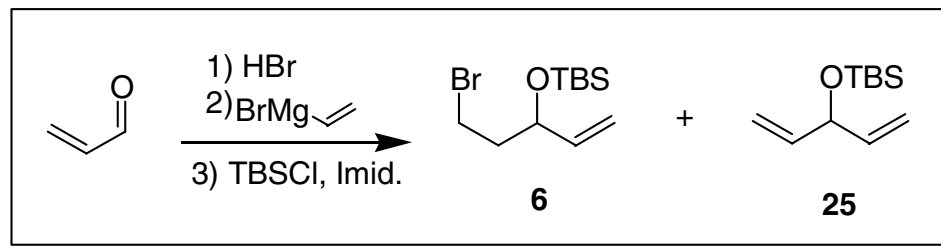

TBS-ethers 6 and 25. A solution of acrolein (20.0 mL, $300 \mathrm{mmol}, 1.0$ equiv) in $\mathrm{Et}_{2} \mathrm{O}(120 \mathrm{~mL})$ at $0^{\circ} \mathrm{C}$ was treated with anhydrous $\mathrm{HBr}$. After NMR had indicated the reaction was complete (ca. $10 \mathrm{~min}$ ), the solution was neutralized with solid $\mathrm{NaHCO}_{3}$ (ca. $3 \mathrm{~g})$ and filtered. The yellow solution was diluted with pentane $(150 \mathrm{~mL})$ and cooled to $78^{\circ} \mathrm{C}$ before adding vinyl magnesium bromide (1.0 M in THF, $300 \mathrm{~mL}, 1.0$ equiv). After $1 \mathrm{~h}$ the reaction was quenched with saturated aqueous $\mathrm{NH}_{4} \mathrm{Cl}$ (ca. $50 \mathrm{~mL}$ ). The mixture was washed with water $(2 \times 100 \mathrm{~mL})$, brine $(100 \mathrm{~mL})$, and dried with $\mathrm{Na}_{2} \mathrm{SO}_{4}$. The solvent was removed in vacuo and the resulting oil taken up in $\mathrm{CH}_{2} \mathrm{Cl}_{2}(500 \mathrm{~mL})$. 
Imidazole (20.4 g, $300 \mathrm{mmol}, 1.0$ equiv) was added followed by TBSCl (45.0 g, 300 mmol, 1.0 equiv) and the mixture was allowed to stir for $16 \mathrm{~h}$. After filtering, the solvent was removed in vacuo and the resulting residue purified by silica gel column chromatography (10:1 pentane: $\left.\mathrm{Et}_{2} \mathrm{O}\right)$ to afford a mixture of bromide 6 (26.8 g, 32\% yield) and olefin 25 (6.5g, 11\% yield) as a colorless oils which could be further purified by low pressure distillation.

TBS-ether 6. b.p. $69-70{ }^{\circ} \mathrm{C} @ 1.5 \mathrm{~mm} \mathrm{Hg} ;{ }^{1} \mathrm{H}$ NMR $\left(500 \mathrm{MHz}, \mathrm{CDCl}_{3}\right) \delta 5.82-$ $5.74(\mathrm{~m}, 1 \mathrm{H}), 5.23-5.18(\mathrm{~m}, 1 \mathrm{H}), 5.10-5.06(\mathrm{~m}, 1 \mathrm{H}), 4.31-4.26(\mathrm{~m}, 1 \mathrm{H}), 3.50-3.38$ (comp $\mathrm{m}, 2 \mathrm{H}), 2.08-1.92$ (comp m, 2H), 0.89 (s, 9H), 0.09 (s, 3H), 0.04 (s, 3H); ${ }^{13} \mathrm{C} \mathrm{NMR}(125$ $\left.\mathrm{MHz}, \mathrm{CDCl}_{3}\right) \delta 140.7,114.9,71.9,41.1,30.1,26.0,18.3,-4.1,-4.7 ; \mathrm{IR}$ (thin film/NaCl) 2955 (s), 2857 (s), 1644 (w), 1472 (m), 1361 (m), 1257 (s), 1162 (w), 1092 (s), 1027 (s), $837(\mathrm{~s}) \mathrm{cm}^{-1}$

TBS-ether 25. b.p. $30-31^{\circ} \mathrm{C} @ 1.5 \mathrm{~mm} \mathrm{Hg} ;{ }^{1} \mathrm{H}$ NMR $\left(500 \mathrm{MHz}, \mathrm{CDCl}_{3}\right) \delta 5.81$ (ddd, $J=5.3,10.5,17.0 \mathrm{~Hz}, 1 \mathrm{H}), 5.22(\mathrm{dt}, J=1.6,17.2 \mathrm{~Hz}, 2 \mathrm{H}), 5.06$ (dt, $J=1.6,16.9 \mathrm{~Hz}$, 2H), 4.63-4.59 (m, 1H), $0.91(\mathrm{~s}, 9 \mathrm{H}), 0.07(\mathrm{~s}, 6 \mathrm{H}) ;{ }^{13} \mathrm{C} \mathrm{NMR}\left(125 \mathrm{MHz}, \mathrm{CDCl}_{3}\right) \delta 140.3$, 113.8, 74.8, 26.0, 18.5, -2.8, -4.6; IR (thin film/NaCl) 2956 (s), 2830 (s), 2858 (s), 1638 (w), $1472(\mathrm{~m}), 1361$ (m), 1254 (s), 1126 (s), 1073 (s), $922(\mathrm{~s}) \mathrm{cm}^{-1}$

\section{Preparation of ester 7}

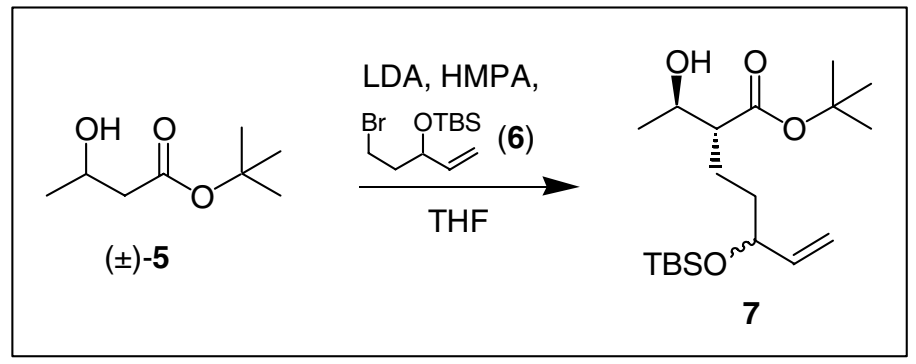

Ester 7. To a solution of ( \pm )-5 (1.00 g, $6.24 \mathrm{mmol}, 1.0$ equiv) in THF (35 mL) and HMPA (3.2 mL, $18.7 \mathrm{mmol}, 3.0$ equiv) at $-40^{\circ} \mathrm{C}$, was added LDA (0.5 M in THF, $26.0 \mathrm{~mL}, 13.1 \mathrm{mmol}, 2.1 \mathrm{equiv})$. After $20 \mathrm{~min}$, a solution of 6 (2.61 g, $9.36 \mathrm{mmol}, 1.5$ equiv) in THF ( $5 \mathrm{~mL}$ ) was added via syringe pump over $1 \mathrm{~h}$. After an additional $2 \mathrm{~h}$, the reaction was warmed to $0^{\circ} \mathrm{C}$ for $1 \mathrm{~h}$ then quenched with saturated aqueous $\mathrm{NaHCO}_{3}(10$ $\mathrm{mL})$. EtOAc $(50 \mathrm{~mL})$ was added and the mixture was washed with water $(25 \mathrm{~mL})$, brine $(25 \mathrm{~mL})$, and dried with $\mathrm{Na}_{2} \mathrm{SO}_{4}$. After the solvent was removed in vacuo, the resulting residue was purified by silica gel column chromatography (9:1 hexanes:EtOAc) to afford ester 7 (1.77 g, 79\% yield) as a colorless oil. (1:1 mixture of diastereomers) ${ }^{1} \mathrm{H}$ NMR $\left(400 \mathrm{MHz}, \mathrm{CDCl}_{3}\right) \delta 5.83-5.71(\mathrm{~m}, 1 \mathrm{H}), 5.14(\mathrm{~d}, J=17.0 \mathrm{~Hz}, 1 \mathrm{H}), 5.03(\mathrm{~d}, J=17.0 \mathrm{~Hz}, 1 \mathrm{H})$ 4.13-4.05 (m, 1H), 3.89-3.80 (m, 1H), 2.62 (br s, 1H), 2.29-2.19 (m, 1H), 1.78-1.40 (comp m, 13H), 1.27-1.15 (comp m, 3H), 0.89 (s, 9H) $0.03(\mathrm{~d}, J=8.6 \mathrm{~Hz}, 6 \mathrm{H}) ;{ }^{13} \mathrm{C} \mathrm{NMR}$ $\left(100 \mathrm{MHz}, \mathrm{CDCl}_{3}\right) \delta 175.1,141.5,141.4,114.2,114.1,81.3,73.6,73.5,68.6,68.5,53.2$, $53.0,35.5,35.4,28.3,26.0,25.2,21.7,21.6,18.4,-4.2,-4.2,-4.7,-4.7$; IR (thin film/NaCl) 3442 (br m), 2957 (m), 2930 (m), 2858 (m), 1728 (m), 1473 (m), 1368 (m), $1254(\mathrm{~m}), 1156(\mathrm{~m}), 1087(\mathrm{~m}) \mathrm{cm}^{-1}$; HRMS (FAB) $\mathrm{m} / z$ found: 359.2618 [calc'd for $\mathrm{C}_{19} \mathrm{H}_{39} \mathrm{O}_{4} \mathrm{Si}(\mathrm{M}+\mathrm{H})$ : 359.2618]. 


\section{Preparation of benzyl ether 8}

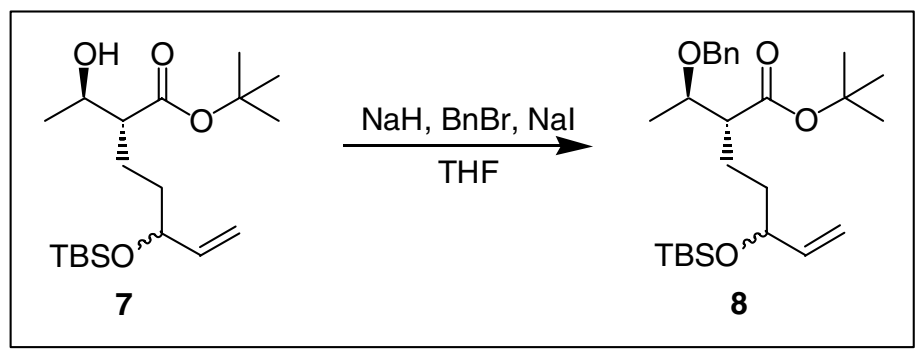

Benzyl ether 8. To a solution of 7 (10.4 g, $28.9 \mathrm{mmol}, 1.0$ equiv) in THF (200 $\mathrm{mL})$ at $0^{\circ} \mathrm{C}$ was added $\mathrm{NaH}(60 \%$ in mineral oil, $1.27 \mathrm{~g}, 31.8 \mathrm{mmol}, 1.1$ equiv) followed by benzyl bromide $(10.3 \mathrm{~mL}, 86.7 \mathrm{mmol}, 3.0$ equiv) and sodium iodide $(1.30 \mathrm{~g}, 8.64$ mmol, 0.3 equiv). The reaction was allowed to warm to $\mathrm{rt}$ and stirred for $12 \mathrm{~h}$ before quenching with water $(100 \mathrm{~mL})$. After diluting with hexanes $(100 \mathrm{~mL})$, the organic layer was separated, washed with brine $(2 \times 100 \mathrm{~mL})$, and dried with $\mathrm{Na}_{2} \mathrm{SO}_{4}$. Removal of the solvent in vасио provided a residue which was purified by silica gel column chromatography (100\% hexanes then 9:1 hexanes:EtOAc) to afford $\mathbf{8}(12.1 \mathrm{~g}, 93 \%$ yield) as a colorless oil. (1:1 mixture of diastereomers) ${ }^{1} \mathrm{H}$ NMR $\left(500 \mathrm{MHz}, \mathrm{CDCl}_{3}\right) \delta 7.33$ 7.19 (comp m, 5H), 5.74 (ddd, $J=5.9,10.5,16.7 \mathrm{~Hz}, 1 \mathrm{H}), 5.14-4.98(\mathrm{~m}, 2 \mathrm{H}), 4.53$ (d, $J=11.4 \mathrm{~Hz}, 1 \mathrm{H}), 4.42(\mathrm{~d}, J=11.2 \mathrm{~Hz}, 1 \mathrm{H}), 4.11-4.03(\mathrm{~m}, 1 \mathrm{H}), 3.69(\mathrm{dq}, J=6.2,8.3 \mathrm{~Hz}$, $1 \mathrm{H}), 2.42-2.33(\mathrm{~m}, 1 \mathrm{H}), 1.59-1.37$ (comp m, 13H), 1.16-1.14 (m, 3H), 0.87-0.86 (m, 9H), 0.03- $-0.03(\mathrm{~m}, 6 \mathrm{H}) ;{ }^{13} \mathrm{C} \mathrm{NMR}\left(125 \mathrm{MHz}, \mathrm{CDCl}_{3}\right) \delta 174.0,173.9,141.6,141.5,128.3$, 127.9, 127.8, 127.5, 114.1, 114.0, 80.4, 80.3, 73.8, 73.4, 71.3, 53.1, 53.0, 35.7, 35.6, 28.3, 26.0, 24.2, 23.8, 18.4, 18.4, 17.1, -4.2, -4.2, -4.7; IR (thin film/NaCl) 2957 (s), 2857 (s), $1728(\mathrm{~s}), 1497(\mathrm{w}), 1472(\mathrm{~m}), 1455(\mathrm{~m}), 1390(\mathrm{~m}), 1366(\mathrm{~s}), 1254(\mathrm{~s}), 1156(\mathrm{~s}) \mathrm{cm}^{-1}$; HRMS (FAB) $\mathrm{m} / z$ found: 449.7186 [calc'd for $\mathrm{C}_{26} \mathrm{H}_{45} \mathrm{O}_{4} \mathrm{Si}(\mathrm{M}+\mathrm{H})$ : 449.7186].

\section{Preparation of alcohol 26}

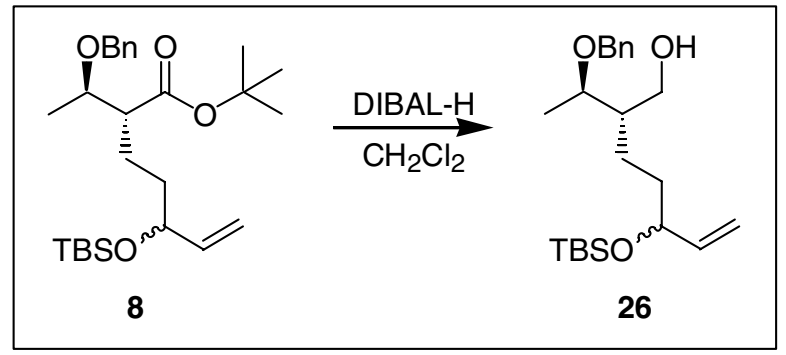

Alcohol 26. A solution of 8 (19.6 g, $43.7 \mathrm{mmol}, 1.0$ equiv) in $\mathrm{CH}_{2} \mathrm{Cl}_{2}(350 \mathrm{~mL})$ at $-78^{\circ} \mathrm{C}$ was treated with DIBAL-H $(17.1 \mathrm{~mL}, 96.2 \mathrm{mmol}, 2.2$ equiv). After $1 \mathrm{~h}$, the reaction was quenched with $20 \%$ aqueous Rochelle's salt $(100 \mathrm{~mL})$. The organic layer was washed with water $(100 \mathrm{~mL})$, brine $(100 \mathrm{~mL})$ and dried with $\mathrm{Na}_{2} \mathrm{SO}_{4}$. After concentration, the resulting residue was purified by silica gel column chromatography (3:1 hexanes:EtOAc) to afford $26(14.9 \mathrm{~g}, 90 \%)$ as a colorless oil. (1:1 mixture of diastereomers) ${ }^{1} \mathrm{H}$ NMR $\left(500 \mathrm{MHz}, \mathrm{CDCl}_{3}\right) \delta$ 7.40-7.25 (comp m, 5H), 5.82-5.72 (m, $1 \mathrm{H}), 5.16-5.11(\mathrm{~m}, 1 \mathrm{H}), 4.65(\mathrm{~d}, J=11.4 \mathrm{~Hz}, 1 \mathrm{H}), 4.41-4.37(\mathrm{~m}, 1 \mathrm{H}), 4.09-4.05(\mathrm{~m}, 1 \mathrm{H})$, 3.86-3.81 (m, 1H), 3.65-3.56 (comp m, 2H), 1.58-1.35 (comp m, 6H), 1.28 (d, J=6.0 Hz, $3 \mathrm{H}), 0.91-0.88$ (comp m, 9H), 0.04 (s, 3H), 0.03 (s, 3H); ${ }^{13} \mathrm{C}$ NMR $\left(125 \mathrm{MHz}, \mathrm{CDCl}_{3}\right) \delta$ 
$141.7,141.6,138.4,128.6,127.9,127.9,127.9,114.0,114.0,79.3,79.2,74.1,73.9,71.2$, 71.2, 63.9, 63.7, 46.3, 46.3, 35.8, 35.7, 26.0, 24.3, 24.1, 18.4, 18.4, 17.8, 17.7, -4.2, -4.7; IR (thin film/NaCl) 3446 (br m), 2954 (m), 2928 (m), 2884 (m), 2856 (m), 1471 (m), $1455(\mathrm{~m}), 1252(\mathrm{~m}), 1088(\mathrm{~m}), 1028(\mathrm{~m}) \mathrm{cm}^{-1}$; HRMS (FAB) $\mathrm{m} / \mathrm{z}$ found: 379.2669 [calc'd for $\mathrm{C}_{22} \mathrm{H}_{39} \mathrm{O}_{2} \mathrm{Si}(\mathrm{M}+\mathrm{H}): 379.2668$ ].

\section{Preparation of aldehyde 9}

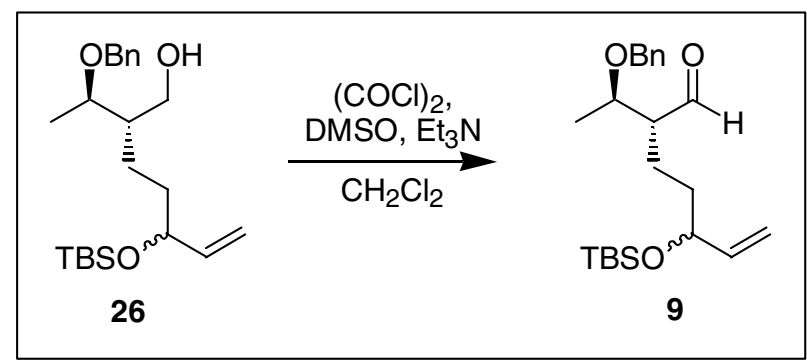

Aldehyde 9. A solution of oxalyl chloride (3.57 mL, $40.9 \mathrm{mmol}, 1.1$ equiv) in $\mathrm{CH}_{2} \mathrm{Cl}_{2}(250 \mathrm{~mL})$ at $-78^{\circ} \mathrm{C}$ was treated with DMSO (5.80 mL, $81.8 \mathrm{mmol}, 2.2$ equiv) and stirred for $10 \mathrm{~min}$. A solution of 26 (14.1 g, $37.2 \mathrm{mmol}, 1.0$ equiv) in $\mathrm{CH}_{2} \mathrm{Cl}_{2}(10 \mathrm{~mL})$ was then added and stirring continued for an additional $10 \mathrm{~min} \mathrm{Et}_{3} \mathrm{~N}(25.9 \mathrm{~mL}, 186$ mmol, 5.0 equiv) was added and the mixture was allowed to warm to rt. Water $(100 \mathrm{~mL})$ was introduced and the organic layer washed with brine $(100 \mathrm{~mL})$ and dried with $\mathrm{Na}_{2} \mathrm{SO}_{4}$. After concentration, the resulting residue was purified by silica gel column chromatography (18:1 then 9:1 hexanes:EtOAc) to afford $9(14.9 \mathrm{~g}, 89 \%)$ as a colorless oil. (1:1 mixture of diastereomers) ${ }^{1} \mathrm{H}$ NMR $\left(500 \mathrm{MHz}, \mathrm{CDCl}_{3}\right) \delta 9.68-9.66(\mathrm{~m}, 1 \mathrm{H})$, 7.36-7.26 (comp m, 5H), 5.80-5.71 (m, 1H), 5.17-5.16 (m, 1H), 5.06-5.04 (m, 1H), 4.62 $(\mathrm{d}, J=11.5 \mathrm{~Hz}, 1 \mathrm{H}), 4.42(\mathrm{~d}, J=11.5 \mathrm{~Hz}, 1 \mathrm{H}), 4.12-4.07(\mathrm{~m}, 1 \mathrm{H}), 3.81(\mathrm{~m}, 1 \mathrm{H}), 2.39-2.33$ (m, 1H), 1.81-1.41 (comp m, 4H), 1.27-1.24 (comp m, 3H), 0.90-0.88 (comp m, 9H), $0.04(\mathrm{~s}, 3 \mathrm{H}), 0.03(\mathrm{~s}, 3 \mathrm{H}) ;{ }^{13} \mathrm{C}$ NMR $\left(125 \mathrm{MHz}, \mathrm{CDCl}_{3}\right) \delta$ 204.7, 204.7, 141.3, 138.4, 128.5, 127.8, 127.8, 114.2, 114.2, 74.9, 74.8, 73.6, 73.4, 70.8, 57.8, 57.7, 35.4, 35.3, 26.0, 21.6, 21.5, 18.3, 17.4, -4.2, -4.7; IR (thin film/NaCl) 2955 (s), 2930 (s), 2857 (s), 1725 (s), 1497 (w), 1472 (m), 1379 (m), 1252 (s), 1091 (s), 836 (s) cm $\mathrm{cm}^{-1}$ HRMS (FAB) $\mathrm{m} / z$ found: 375.2355 [calc'd for $\mathrm{C}_{22} \mathrm{H}_{35} \mathrm{O}_{3} \mathrm{Si}(\mathrm{M}-\mathrm{H})$ : 375.2355 ].

\section{Preparation of triene 4}

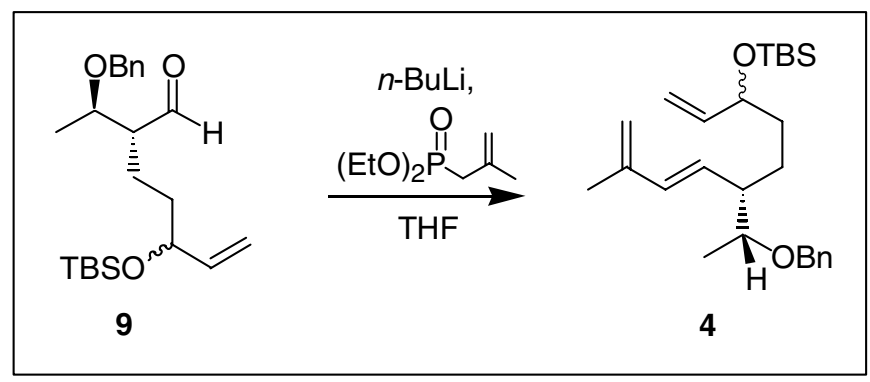

Triene 4. A solution of methallyl phosphonic acid diethyl ester (6.92 g, 36.0 mmol, 1.3 equiv) in THF $(300 \mathrm{~mL})$ at $-78^{\circ} \mathrm{C}$ was treated with $n$-BuLi $(2.3 \mathrm{M}$ in hexanes, $15.7 \mathrm{~mL}, 36.0 \mathrm{mmol}, 1.3$ equiv) and the solution was stirred for $5 \mathrm{~min}$. A solution of 9 (10.4 g, $27.7 \mathrm{mmol}, 1.0$ equiv) in THF (10 mL) was added and stirring continued for an additional $15 \mathrm{~min}$. The reaction was warmed to $\mathrm{rt}$ and stirred for an additional $1 \mathrm{~h}$ before 
quenching with water $(100 \mathrm{~mL})$. The mixture was diluted with hexanes $(200 \mathrm{~mL})$ and the organic layer was washed with water $(3 \mathrm{x} 100 \mathrm{~mL})$, brine $(100 \mathrm{~mL})$, and dried with $\mathrm{Na}_{2} \mathrm{SO}_{4}$. Concentration in vacuo afforded $4(24.9 \mathrm{~g}, 90 \%)$ as a colorless oil which could be advanced without further purification. An analytical sample, however, could be obtained by silica gel column chromatography $\left(9: 1\right.$ hexanes:EtOAc, $\left.1 \% \mathrm{Et}_{3} \mathrm{~N}\right)$. (1:1 mixture of diastereomers) ${ }^{1} \mathrm{H}$ NMR $\left(500 \mathrm{MHz}, \mathrm{CDCl}_{3}\right) \delta 7.40-7.25$ (comp m, 5H), 6.13 $(\mathrm{d}, J=15.4 \mathrm{~Hz}, 1 \mathrm{H}), 5.82-5.74(\mathrm{~m}, 1 \mathrm{H}), 5.54-5.41(\mathrm{~m}, 1 \mathrm{H}), 5.16-5.10(\mathrm{~m}, 1 \mathrm{H}), 5.04-4.99$ $(\mathrm{m}, 1 \mathrm{H}), 4.89$ (s, 2H), $4.58(\mathrm{~d}, J=11.2 \mathrm{~Hz}, 1 \mathrm{H}), 4.45(\mathrm{~d}, J=11.9 \mathrm{~Hz}, 1 \mathrm{H}), 4.10-4.05$ (m, $1 \mathrm{H}), 3.53-3.48(\mathrm{~m}, 1 \mathrm{H}), 2.21-2.12(\mathrm{~m}, 1 \mathrm{H}), 1.85-1.83(\mathrm{~m}, 3 \mathrm{H}), 1.70-1.25$ (comp m, 4H), 1.16-1.12 (comp m, 3H), 0.90 (s, 9H), 0.05-0.03 (comp m, 6H); ${ }^{13} \mathrm{C}$ NMR (125 MHz, $\left.\mathrm{CDCl}_{3}\right) \delta 142.3,142.0,141.8,139.3,138.5,134.9,134.9,131.5,128.6,128.4,128.4$, $127.9,127.8,127.8,127.7,127.5,114.7,114.7,113.7,113.6,78.0,77.9,74.1,72.3,70.9$, 70.8, 49.1, 49.0, 48.9, 36.3, 36.2, 26.4, 26.3, 26.1, 18.9, 18.4, 17.2, 17.1, -4.2, -4.2, -4.6; IR (thin film/NaCl) 2955 (m), 2929 (m), 2857 (m), 1472 (w), 1454 (w), 1361 (m), 1252 (m), $1090(\mathrm{~m}), 1028(\mathrm{~m}), 836(\mathrm{~m}) \mathrm{cm}^{-1}$; HRMS (FAB) $\mathrm{m} / \mathrm{z}$ found: 415.7039 [calc'd for $\left.\mathrm{C}_{26} \mathrm{H}_{43} \mathrm{O}_{2} \mathrm{Si}(\mathrm{M}+\mathrm{H}): 415.7039\right]$.

\section{Preparation of cis-decalin 10}

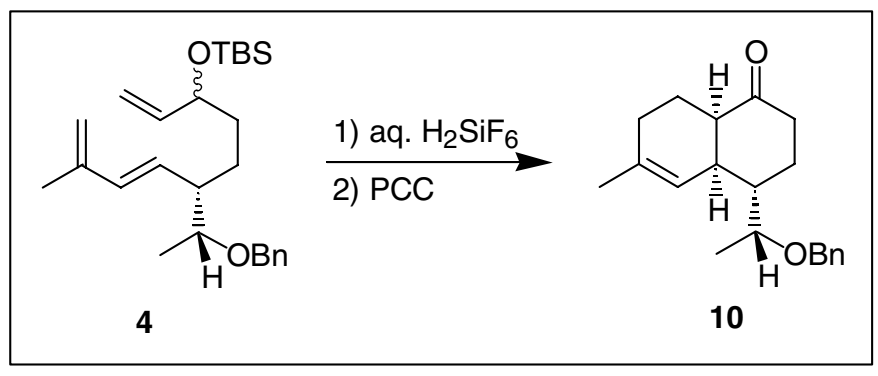

Note: The diastereoselectivity of the cyclization correlated with the Lewis acidity of the oxidant. Lewis acidic oxidants $\left(\mathrm{Cr}^{6+}\right.$ and $\left.\mathrm{Mn}^{4+}\right)$ afforded high to moderate diastereoselectivity, while non-metal based oxidants (Swern conditions) led to low diastereoselectivity.

\begin{tabular}{|c|c|}
\hline Conditions & Diastereoselectivity \\
\hline $\begin{array}{c}\text { Jones ox. } \\
\left(\mathrm{H}_{2} \mathrm{SO}_{4}+\mathrm{Cr}^{6+}\right)\end{array}$ & $9: 1$ \\
\hline $\mathrm{MnO}_{2}\left(\mathrm{Mn}^{4+}\right)$ & $6: 1$ \\
\hline $\mathrm{PCC}_{\left(\mathrm{Cr}^{6+}\right)}$ & $5: 1$ \\
\hline Swern ox. & $3: 1$ \\
\hline
\end{tabular}

cis-Decalin 10. A solution of 4 (6.92 g, $22.0 \mathrm{mmol}, 1.0$ equiv) in $\mathrm{MeCN}$ (225 $\mathrm{mL}$ ) at $0^{\circ} \mathrm{C}$ was treated with $20 \%$ aqueous fluorosilicic acid $(1.6 \mathrm{~mL}, 2.2 \mathrm{mmol}, 0.1$ equiv). After ca. $4 \mathrm{~h}$, the reaction was quenched with $\mathrm{K}_{2} \mathrm{CO}_{3}$ (ca. $5 \mathrm{~g}$ ) and diluted with hexanes $(100 \mathrm{~mL})$ and water $(100 \mathrm{~mL})$. The organic layer was washed with water (100 $\mathrm{mL})$, brine $(100 \mathrm{~mL})$ and dried with $\mathrm{Na}_{2} \mathrm{SO}_{4}$. Concentration in vacuo provided a residue which was taken up in $\mathrm{CH}_{2} \mathrm{Cl}_{2}(225 \mathrm{~mL})$. The solution was cooled to $0^{\circ} \mathrm{C}$ and $\mathrm{PCC}(19.0$ 
g, 88.0 mmol, 4.0 equiv) was added. After $1 \mathrm{~h}$, the reaction was warmed to $\mathrm{rt}$ and stirred for an additional $12 \mathrm{~h}$. Celite (ca $10 \mathrm{~g}$ ) was added and the mixture was filtered. Concentration in vacuo followed by silica gel column chromatography $(9: 1$ hexanes:EtOAc) afforded 10 (4.73 g, 72\% yield from 4) as a colorless oil. ${ }^{1} \mathrm{H}$ NMR (400 $\mathrm{MHz}_{\mathrm{CDCl}}$ ) $\delta$ 7.28-7.18 (comp m, 5H), 5.16-5.13 (m, 1H), 4.59 (d, J=12.1 Hz, 1H), $4.38(\mathrm{~d}, J=12.1 \mathrm{~Hz}, 1 \mathrm{H}), 3.79-3.71(\mathrm{~m}, 1 \mathrm{H}), 2.64$ (br s, 1H), 2.24-2.15 (comp m, 3H), 2.05-1.75 (comp m, 5H), 1.61-1.52 (comp m, 4H), 1.43-1.35 (m, 1H), 1.18 (d, J=6.2 Hz, $3 \mathrm{H}) ;{ }^{13} \mathrm{C}$ NMR $\left(100 \mathrm{MHz}, \mathrm{CDCl}_{3}\right) \delta 214.0,138.8,135.6,128.5,127.9,127.7,124.0$, 74.1, 70.8, 46.2, 44.3, 38.1, 37.9, 28.1, 23.8, 23.8, 22.9, 15.8; IR (thin film/NaCl) 2926 (m), 2876 (m), 1707 (s), 1496 (w), 1452 (m), 1376 (m), 1323 (w), 1121 (m), 1090 (m), $1073(\mathrm{~m}) \mathrm{cm}^{-1}$; HRMS (FAB) $\mathrm{m} / \mathrm{z}$ found: 299.2011 [calc'd for $\mathrm{C}_{20} \mathrm{H}_{27} \mathrm{O}_{2}(\mathrm{M}+\mathrm{H})$ : 299.2011].

\section{Preparation of epoxide 27}

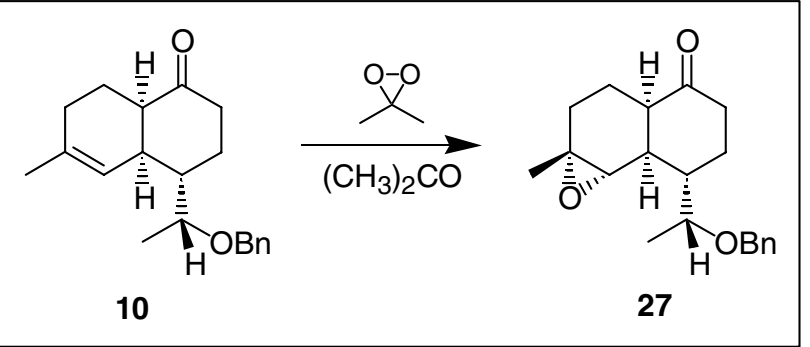

Epoxide 27. To a $0.09 \mathrm{M}$ solution of dimethyl dioxirane in acetone $(94.3 \mathrm{~mL}$, $8.49 \mathrm{mmol}, 1.5$ equiv) at $-78^{\circ} \mathrm{C}$, was added olefin 10 (1.69 g, $5.65 \mathrm{mmol}, 1.0$ equiv) in acetone $(10 \mathrm{~mL})$. After $2 \mathrm{~h}$, the solution was slowly warmed to $25^{\circ} \mathrm{C}$ before removing the solvent in vacuo. NMR of the resulting oil showed a mixture of diastereomers in the ratio of 94:6 which could be purified by silica gel column chromatography (9:1 then 3:1 hexanes:EtOAc) to afford 27 (1.51 g, 85\% yield) as a colorless oil. ${ }^{1} \mathrm{H}$ NMR $(500 \mathrm{MHz}$, $\left.\mathrm{CDCl}_{3}\right) \delta$ 7.26-7.17 (comp m, 5H), $4.61(\mathrm{~d}, J=11.7 \mathrm{~Hz}, 1 \mathrm{H}), 4.35(\mathrm{~d}, J=11.9 \mathrm{~Hz}, 1 \mathrm{H})$, 3.65-3.59 (m, 1H), $2.59(\mathrm{~s}, 1 \mathrm{H}), 2.57-2.53(\mathrm{~m}, 1 \mathrm{H}), 2.24-2.16(\mathrm{~m}, 1 \mathrm{H}), 2.13-2.01$ (comp $\mathrm{m}, 2 \mathrm{H}), 1.84-1.66$ (comp m, 6H), 1.23-1.13 (comp m, 7H); ${ }^{13} \mathrm{C} \mathrm{NMR} \mathrm{(125} \mathrm{MHz,} \mathrm{CDCl}_{3}$ ) $\delta 212.8,138.5,128.6,128.1,127.9,74.6,70.9,63.9,58.1,43.8,42.0,38.1,37.0,25.9$, 23.6, 23.5, 19.2, 16.3; IR (thin film/NaCl) 2957 (m), 2928 (m), 2872 (m), 1707 (s), 1495 (w), 1453 (m), 1421 (w), 1378 (m), 1330 (w), 1090 (m) $\mathrm{cm}^{-1}$; HRMS (FAB) $\mathrm{m} / z$ found: 315.1959 [calc'd for $\mathrm{C}_{20} \mathrm{H}_{27} \mathrm{O}_{3}(\mathrm{M}+\mathrm{H}): 315.1959$ ].

\section{Preparation of olefin 11}

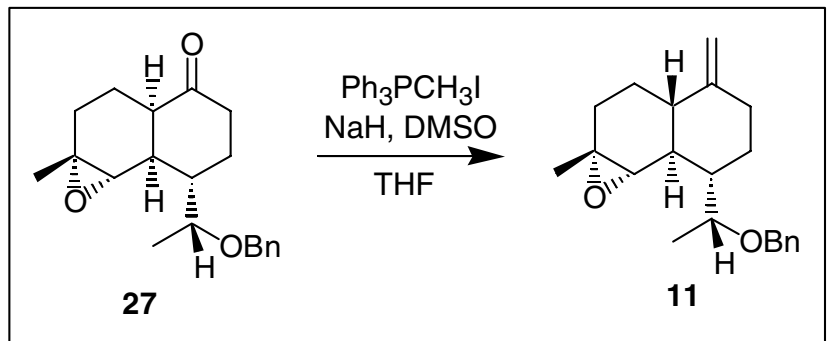

Olefin 11. To a solution of epoxide 27 (1.51 g, $4.80 \mathrm{mmol}, 1.0$ equiv) in $\mathrm{MeOH}$ $(50 \mathrm{~mL})$ at $\mathrm{rt}$ was added $\mathrm{NaOMe}(15 \mathrm{mg}, 0.24 \mathrm{mmol}, 0.05$ equiv). After $36 \mathrm{~h}$, the 
solution was concentrated under reduced pressure, diluted with $\mathrm{CH}_{2} \mathrm{Cl}_{2}(100 \mathrm{~mL})$, and washed with water $(2 \times 25 \mathrm{~mL})$ and brine $(25 \mathrm{~mL})$. After drying with $\mathrm{Na}_{2} \mathrm{SO}_{4}$, concentration in vacuo gave a 3:2 equilibrium mixture of decalins, which could be used without further purification. A solution of DMSO $(6.8 \mathrm{~mL}, 96.0 \mathrm{mmol}, 20$ equiv) in THF $(20 \mathrm{~mL})$ was treated with $\mathrm{NaH}$ (60\% in mineral oil, $394 \mathrm{mg}, 9.60 \mathrm{mmol}, 2.0$ equiv). The resulting slurry was heated at $60^{\circ} \mathrm{C}$ for $2 \mathrm{~h}$ then cooled to $25{ }^{\circ} \mathrm{C}$ before adding methyltriphenylphosphonium iodide (3.43 g, $9.60 \mathrm{mmol}, 2.0$ equiv) and THF (10 mL). The resulting yellow mixture was added in 0.1 equiv portions every $2 \mathrm{~h}$ to a crude solution of decalins from 27 in THF $(50 \mathrm{~mL})$. After reaction was complete by TLC, it was quenched with water $(50 \mathrm{~mL})$ and diluted with hexanes $(100 \mathrm{~mL})$. The aqueous layer was separated and extracted with hexanes $(2 \times 25 \mathrm{~mL})$ and the combined organic fractions were washed with water $(3 \times 50 \mathrm{~mL})$, dried $\left(\mathrm{Na}_{2} \mathrm{SO}_{4}\right)$, and concentrated. The resulting residue was purified by silica gel column chromatography $(100 \%$ hexanes then 9:1 hexanes:EtOAc) to give $11\left(1.23 \mathrm{~g}, 82 \%\right.$ from 27) as a colorless oil. ${ }^{1} \mathrm{H}$ NMR (500 $\left.\mathrm{MHz} \mathrm{CDCl}_{3}\right) \delta$ 7.29-7.16 (comp m, 5H), 4.59 (s, 1H), 4.47 (q, J=11.1 Hz, 1H), 4.42 (s, $1 \mathrm{H}), 3.96-3.90(\mathrm{~m}, 1 \mathrm{H}), 2.77(\mathrm{~s}, 1 \mathrm{H}), 2.31-2.26(\mathrm{~m}, 1 \mathrm{H}), 2.04-1.85$ (comp m, 4H), 1.631.43 (comp m, 3H), 1.26-1.13 (comp m, 7H), 1.08 (d, J=6.3 Hz, 3H); ${ }^{13} \mathrm{C}$ NMR (100 $\left.\mathrm{MHz} \mathrm{CDCl}_{3}\right) \delta 151.9,139.1,128.5,127.6,127.6,104.8,74.4,70.5,60.9,58.4,45.9$, 43.6, 43.2, 35.8, 30.3, 27.3, 23.7, 22.5, 14.3; IR (thin film/NaCl) $2978(\mathrm{~m}), 2930(\mathrm{~m})$, 2880 (m), 1648 (m), 1453 (m), 1379 (m), 1135 (m), $1101(\mathrm{~m}), 1071(\mathrm{~m}), 887(\mathrm{~m}) \mathrm{cm}^{-1}$; HRMS (FAB) $m / z$ found: 313.2169 [calc'd for $\mathrm{C}_{21} \mathrm{H}_{29} \mathrm{O}_{2}(\mathrm{M}+\mathrm{H}): 313.2168$ ].

\section{Preparation of alcohols 12 and 28}
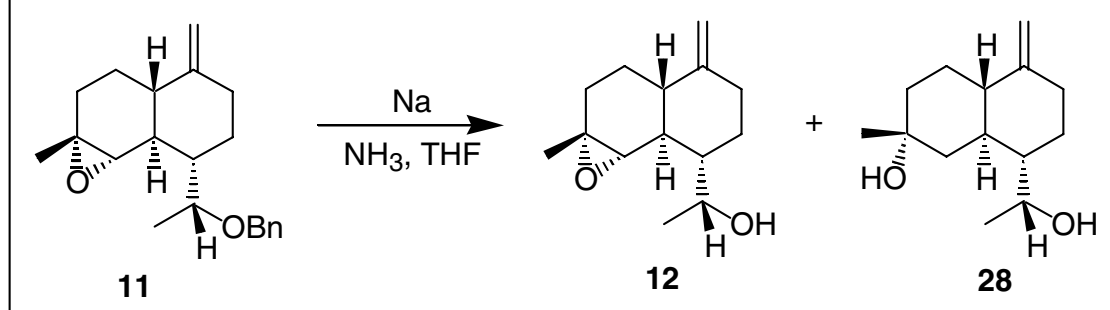

Alcohols 12 and 28 . To condensed ammonia $(40 \mathrm{~mL})$ at $-78^{\circ} \mathrm{C}$ was added sodium (368 mg, $16 \mathrm{mmol}, 10.0$ equiv) followed by 11 (500 mg, $1.60 \mathrm{mmol}, 1.0$ equiv), which was contaminated with ca. $1 \%$ of the corresponding cis-decalin, in THF (4 mL). After $1 \mathrm{~h}$, the reaction was quenched with solid ammonium chloride and the ammonia allowed to evaporate. The resulting residue was taken up in EtOAc $(50 \mathrm{~mL})$, washed with water $(2 \times 25 \mathrm{~mL})$, brine $(25 \mathrm{~mL})$ and dried with $\mathrm{Na}_{2} \mathrm{SO}_{4}$. After concentration, the resulting oil was purified by silica gel column chromatography (3:1 then 1:1 hexanes:EtOAc) to afford $\mathbf{1 2}(324 \mathrm{mg}, 91 \%)$ and $\mathbf{2 8}(7 \mathrm{mg}, 2 \%)$ as a white solid.

Alcohol 12. ${ }^{1} \mathrm{H}$ NMR $\left(400 \mathrm{MHz}, \mathrm{CDCl}_{3}\right) \delta 4.66(\mathrm{~s}, 1 \mathrm{H}), 4.50(\mathrm{~s}, 1 \mathrm{H}), 4.38-4.32$ $(\mathrm{m}, 1 \mathrm{H}), 2.98(\mathrm{~s}, 1 \mathrm{H}), 2.40-2.34(\mathrm{~m}, 1 \mathrm{H}), 2.12-1.95(\mathrm{comp} \mathrm{m}, 3 \mathrm{H}), 1.83-1.76(\mathrm{~m}, 1 \mathrm{H})$, 1.71-1.51 (comp m, 4H), 1.32-1.12 (comp m, 9H); ${ }^{13} \mathrm{C} \mathrm{NMR} \mathrm{(100} \mathrm{MHz,} \mathrm{CDCl}_{3}$ ) $\delta 151.6$, $104.9,67.3,60.9,58.4,46.1,45.8,43.5,35.6,30.2,26.8,23.7,22.4,17.7$; IR (thin film/NaCl) 3437 (br m), 2975 (m), 2931 (m), 2878 (m), 1649 (m), 1447 (m), 1422 (w), $1379(\mathrm{~m}), 1100(\mathrm{~m}), 888(\mathrm{~m}) \mathrm{cm}^{-1}$; HRMS (FAB) $\mathrm{m} / \mathrm{z}$ found: 223.1699 [calc'd for $\left.\mathrm{C}_{14} \mathrm{H}_{23} \mathrm{O}_{2}(\mathrm{M}+\mathrm{H}): 223.1698\right]$. 
Alcohol 28. m.p. $154-156{ }^{\circ} \mathrm{C}$ (recrystallized from 3:1 hexanes:EtOAc); ${ }^{1} \mathrm{H}$ NMR $\left(400 \mathrm{MHz}, \mathrm{CDCl}_{3}\right) \delta 4.69(\mathrm{~s}, 1 \mathrm{H}), 4.60(\mathrm{~s}, 1 \mathrm{H}), 4.16-4.09(\mathrm{~m}, 1 \mathrm{H}), 2.43(\mathrm{dt}, J=3.3,12.8$ $\mathrm{Hz}, 1 \mathrm{H}$ ), 2.08-1.93 (comp m, 2H), 1.82-1.38 (comp m, 8H), 1.33-1.02 (comp m, 10H); ${ }^{13} \mathrm{C}$ NMR $\left(125 \mathrm{MHz}, \mathrm{CDCl}_{3}\right) \delta 152.3,104.8,69.6,67.2,49.0,45.8,43.1,41.2,38.2,36.2$, 31.8, 25.9, 24.4, 16.8; IR (thin film/NaCl) 3372 (m), 3081 (w), 2964 (m), 2929 (m), 2845 (m), 1644 (m), 1443 (m), 1374 (m), 1309 (w), 1264 (w) $\mathrm{cm}^{-1}$; HRMS (FAB) m/z found: 206.1668 [calc'd for $\mathrm{C}_{14} \mathrm{H}_{22} \mathrm{O}\left(\mathrm{M}+-\mathrm{H}_{2} \mathrm{O}\right)$ : 206.1671].

\section{Preparation of ketone 3}

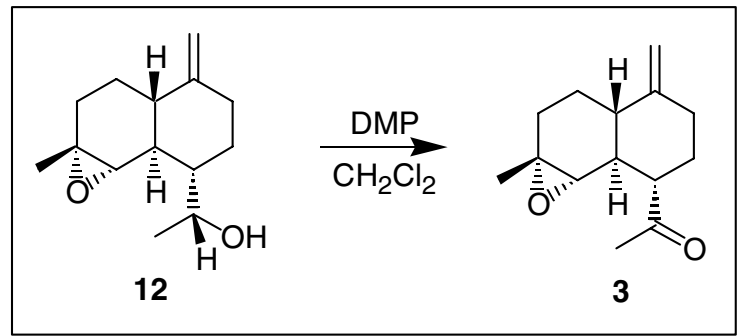

Ketone 3. A solution of alcohol $12(300 \mathrm{mg}, 1.35 \mathrm{mmol}, 1.0$ equiv) in undistilled $\mathrm{CH}_{2} \mathrm{Cl}_{2}(20 \mathrm{~mL})$ was treated with Dess-Martin periodinane $(744 \mathrm{mg}, 1.76 \mathrm{mmol}, 1.3$ equiv) and the mixture was stirred at $0^{\circ} \mathrm{C}$ for $2 \mathrm{~h}$. Saturated aqueous $\mathrm{NaHCO}_{3}(5 \mathrm{~mL})$ and $\mathrm{Na}_{2} \mathrm{~S}_{2} \mathrm{O}_{3}(5 \mathrm{~mL})$ were then added. After $30 \mathrm{~min}$, the organic layer was separated and dried with $\mathrm{Na}_{2} \mathrm{SO}_{4}$. Concentration in vacuo gave a residue which was purified by silica gel column chromatography (9:1 then 3:1 hexanes:EtOAc) to afford ketone 3 (253 $\mathrm{mg}$, $85 \%$ yield) as a white, amorphous solid. ${ }^{1} \mathrm{H} \mathrm{NMR}\left(400 \mathrm{MHz}, \mathrm{CDCl}_{3}\right) \delta 4.72(\mathrm{~d}, J=1.1$ $\mathrm{Hz}, 1 \mathrm{H}), 4.57$ (d, J=1.1 Hz, 1H), 2.57 (dt, $J=12.0,3.4 \mathrm{~Hz}, 1 \mathrm{H}), 2.50$ (s, 1H), 2.44-2.39 (m, 1H), $2.21(\mathrm{~s}, 3 \mathrm{H}), 2.15-2.00$ (comp m, 3H), 1.78 (t, J=12.0 Hz, 1H), 1.72-1.57 (comp $\mathrm{m}, 2 \mathrm{H}), 1.53-1.42$ (comp m, 2H), 1.36 (dt, $J=4.6,11.7 \mathrm{~Hz}, 1 \mathrm{H}), 1.29$ (s, 3H); ${ }^{13} \mathrm{C}$ NMR $\left(100 \mathrm{MHz}, \mathrm{CDCl}_{3}\right) \delta 211.4,149.9,106.2,61.9,58.3,56.2,44.8,42.8,35.4,31.7,30.3$, 27.9, 23.4, 22.0; IR (thin film/NaCl) 2973 (w), 2932 (m), 2887 (w), 2853 (w), 1706 (m), 1650 (w), 1440 (w), 1424 (w), 1374 (w), 1183 (w) $\mathrm{cm}^{-1}$; HRMS (FAB) $\mathrm{m} / \mathrm{z}$ found: 221.1543 [calc'd for $\mathrm{C}_{14} \mathrm{H}_{21} \mathrm{O}_{2}(\mathrm{M}+\mathrm{H}): 221.1542$ ].

\section{Preparation of alcohols 13 and 12}

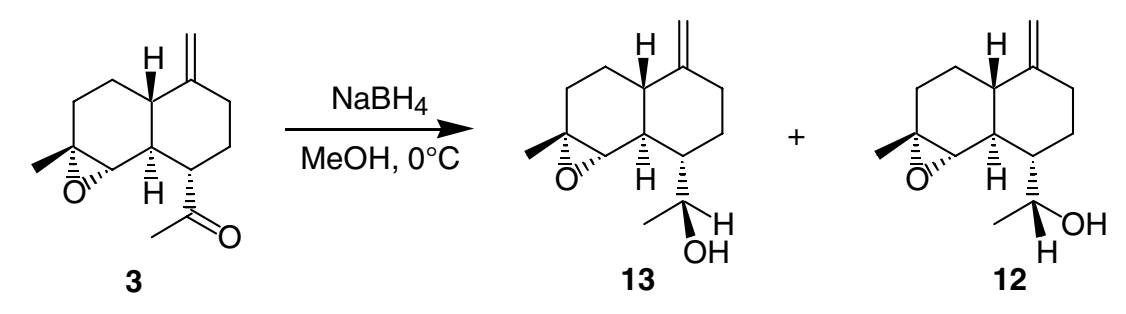

Alcohols 13 and 12. To a solution of $\mathbf{3}(15 \mathrm{mg}, 0.068 \mathrm{mmol}, 1.0$ equiv) in $\mathrm{MeOH}$ $(5 \mathrm{~mL})$ at $0^{\circ} \mathrm{C}$ was added $\mathrm{NaBH}_{4}(8 \mathrm{mg}, 0.204 \mathrm{mmol}, 3.0$ equiv) in one portion. After 30 min, EtOAc $(25 \mathrm{~mL})$ was added and the mixture was washed with water $(25 \mathrm{~mL})$, brine (25 mL), and dried with $\mathrm{Na}_{2} \mathrm{SO}_{4}$. Following concentration under reduced pressure, NMR analysis of the crude residue revealed a mixture of alcohols in the ratio of ca. 91:9. Following purification of the mixture by silica gel column chromatography $(3: 1$ 
hexanes:EtOAc), the major compound was revealed to be alcohol 13 (19 $\mathrm{mg}, 77 \%$ ), a colorless oil, and the minor compound was identical with material obtained from benzyl deprotection of $\mathbf{1 1}$ and was determined to be $\mathbf{1 2}$.

Alcohol 13. ${ }^{1} \mathrm{H}$ NMR (400 MHz, $\left.\mathrm{CDCl}_{3}\right) \delta 4.66(\mathrm{~s}, 1 \mathrm{H}), 4.50(\mathrm{~s}, 1 \mathrm{H}), 4.30-4.22$ (m, 1H), $3.17(\mathrm{~s}, 1 \mathrm{H}), 2.39-2.33(\mathrm{~m}, 1 \mathrm{H}), 2.14-1.97$ (comp m, 2H), 1.90-1.82 (m, 1H), 1.71-1.43 (comp m, 6H), 1.40-1.21 (comp m, 8H); $\left.{ }^{13} \mathrm{C} \mathrm{NMR} \mathrm{(100} \mathrm{MHz,} \mathrm{CDCl}_{3}\right) \delta 151.5$, $104.9,67.3,61.2,58.6,45.7,44.6,43.3,35.7,30.3,27.7,23.8,22.6,20.5$; IR (thin film/NaCl) 3440 (s), 2974 (s), 2932 (s), 1649 (m), 1448 (m), 1422 (w), 1378 (m), 1134 (w), 1011 (w), 887 (s) $\mathrm{cm}^{-1}$; HRMS (FAB) $\mathrm{m} / \mathrm{z}$ found: 223.1698 [calc'd for $\mathrm{C}_{14} \mathrm{H}_{23} \mathrm{O}_{2}$ $(\mathrm{M}+\mathrm{H}): 223.1698]$.

\section{Preparation of ester 16}

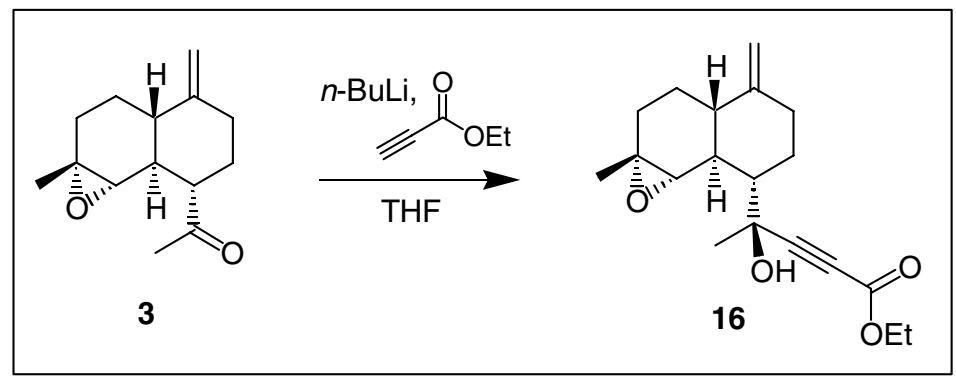

Ester 16. A solution of ethyl propiolate $(28 \mu \mathrm{L}, 0.272 \mathrm{mmol}, 4.0$ equiv) in THF $(3 \mathrm{~mL})$ at $-78^{\circ} \mathrm{C}$ was treated with $\mathrm{n}$-BuLi $(2.3 \mathrm{M}$ in hexanes, $117 \mu \mathrm{L}, 0.27 \mathrm{mmol}, 4.0$ equiv). After $5 \mathrm{~min}, 3$ (15 mg, $0.068 \mathrm{mmol}, 1.0$ equiv) in THF (1 mL) was added. The temperature was maintained at $-78^{\circ} \mathrm{C}$ for 30 min before slowly warming to $0^{\circ} \mathrm{C}$ for an additional $1 \mathrm{hr}$. Water $(5 \mathrm{~mL})$ was added followed by EtOAc $(15 \mathrm{~mL})$. The layers were separated and the aqueous portion was extracted with EtOAc $(10 \mathrm{~mL})$. The combined organic fractions were washed with brine $(15 \mathrm{~mL})$ and dried with $\mathrm{Na}_{2} \mathrm{SO}_{4}$ before concentrating in vacuo. Crude NMR showed ca. 98:2 mixture of diastereomers which could be purified by silica gel column chromatography (3:1 hexanes:EtOAc) to afford 16 $(19 \mathrm{mg}, 89 \%)$ as a colorless oil. ${ }^{1} \mathrm{H} \mathrm{NMR}\left(500 \mathrm{MHz}, \mathrm{CDCl}_{3}\right) \delta 4.68(\mathrm{~s}, 1 \mathrm{H}), 4.54(\mathrm{~s}, 1 \mathrm{H})$, 4.24 (q, J=7.2 Hz, 2H), 3.93 (s, 1H), 2.42 (s, 1H), 2.39 (dt, J=4.5, 13.1 Hz, 1H), 2.352.29 (m, 1H), 2.14-1.98 (comp m, 3H), 1.73-1.51 (comp m, 7H), 1.41-1.17 (comp m 8H); ${ }^{13} \mathrm{C}$ NMR $\left(125 \mathrm{MHz}, \mathrm{CDCl}_{3}\right) \delta 153.7,151.0,105.2,90.9,77.0,72.6,62.3,61.1,59.0$, 48.8, 45.7, 43.0, 35.7, 32.3, 30.2, 25.0, 23.6, 22.4, 14.2; IR (thin film/ $/ \mathrm{NaCl}$ ) 3400 (br m), 2984 (m), 2936 (m), 2237 (m), 1712 (s), 1650 (m), 1447 (m), 1368 (m), 1246 (s), 1092 (m) $\mathrm{cm}^{-1}$; HRMS (EI) $\mathrm{m} / z$ found: 318.1835 [calc'd for $\mathrm{C}_{19} \mathrm{H}_{26} \mathrm{O}_{4}(\mathrm{M}+)$ : 318.1835 ].

\section{Preparation of aziridines 17 and 29}

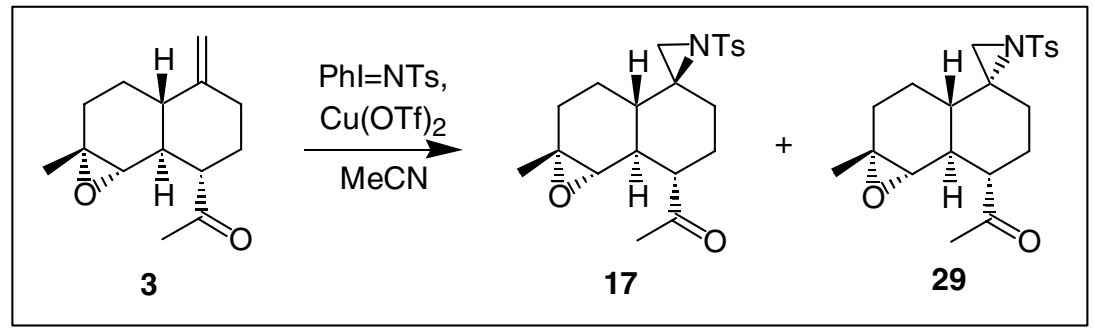


Aziridines 17 and 29. A solution of ketone 3 (1.06 g, $4.80 \mathrm{mmol}, 1.0$ equiv) in dry $\mathrm{MeCN}(250 \mathrm{~mL})$ with $4 \AA$ sieves (ca. $3 \mathrm{~g}$ ) at $25^{\circ} \mathrm{C}$, was treated with $\mathrm{N}$ tosyliminophenyliodinane $\left(3.59 \mathrm{~g}, 9.62 \mathrm{mmol}, 2.0\right.$ equiv). After 2 minutes, $\mathrm{Cu}(\mathrm{OTf})_{2}(87$ $\mathrm{mg}, 0.24 \mathrm{mmol}, 0.05$ equiv) was added and the mixture was vigorously stirred for $8 \mathrm{~h}$. The mixture was then diluted with EtOAc $(50 \mathrm{~mL})$ and filtered through a short plug of silica gel. After removing the solvent in vacuo, the resulting residue, ca. 5:1 mixture of aziridine diastereomers, was purified by silica gel column chromatography $(3: 1$ hexanes:EtOAc) to afford aziridine 17 (1.12 g, 60\% yield) as a white solid. Mixed fractions were analyzed by HPLC to give an analytical sample of $\mathbf{2 9}$ (37 $\mathrm{mg}, 2 \%$ yield) as a white, amorphous solid.

Aziridine 17. m.p. $195-197^{\circ} \mathrm{C}$ (recrystallized from $3: 1$ hexanes:EtOAc); ${ }^{1} \mathrm{H}$ NMR $\left(500 \mathrm{MHz}, \mathrm{CDCl}_{3}\right) \delta 7.80(\mathrm{~d}, J=8.5 \mathrm{~Hz}, 2 \mathrm{H}), 7.32(\mathrm{~d}, J=8.6 \mathrm{~Hz}, 2 \mathrm{H}), 2.61$ (dt, $J=3.7,12.1 \mathrm{~Hz}, 1 \mathrm{H}), 2.54$ (s, 1H), 2.48 (s, 1H), 2.44 (s, 3H), 2.42-2.28 (comp m, 2H), $2.26(\mathrm{~s}, 3 \mathrm{H}), 2.23-2.17$ (comp m, 2H), 2.01-1.96 (m, 1H), 1.93 (t, J=11.8 Hz, 1H), 1.651.43 (comp m, 4H), 1.28 (s, 3H), 0.74 (dq, J=4.4, $12.4 \mathrm{~Hz}, 1 \mathrm{H}) ;{ }^{13} \mathrm{C}$ NMR $(100 \mathrm{MHz}$, $\left.\mathrm{CDCl}_{3}\right) \delta 210.5,144.1,137.9,129.7,127.4,61.5,58.6,54.5,54.0,42.2,42.2,36.2,30.6$, 29.9, 29.7, 29.0, 23.2, 21.7, 18.1; IR (thin film/NaCl) 3269 (br m), 2928 (m), 2253 (w), 1708 (s), 1598 (m), 1451 (m), 1319 (s), 1159 (s), 1088 (m), 1002 (m) cm ${ }^{-1}$; HRMS (EI) $\mathrm{m} / \mathrm{z}$ found: 389.1661 [calc'd for $\mathrm{C}_{21} \mathrm{H}_{27} \mathrm{NO}_{4} \mathrm{~S}(\mathrm{M}+)$ : 389.1661 ].

Aziridine 29. ${ }^{1} \mathrm{H}$ NMR $\left(500 \mathrm{MHz}, \mathrm{CDCl}_{3}\right) \delta 7.84(\mathrm{~d}, J=7.1 \mathrm{~Hz}, 2 \mathrm{H}), 7.32(\mathrm{~d}$, $J=7.9 \mathrm{~Hz}, 2 \mathrm{H}$ ), 2.55-2.41 (comp m, 8H), 2.38-2.27 (m, 1H), 2.27-2.17 (comp m, 4H), 2.10-1.93 (comp m, 3H), 1.60-1.51 (m, 1H), 1.33-1.23 (comp m, 4H), 1.12 (dq, J=4.4, $12.1 \mathrm{~Hz}, 1 \mathrm{H}), 0.98(\mathrm{~m}, 1 \mathrm{H}) ;{ }^{13} \mathrm{C} \mathrm{NMR}\left(125 \mathrm{MHz} \mathrm{CDCl}_{3}\right) \delta 211.6,144.2,137.6,129.7$, 127.7, 61.3, 58.2, 56.4, 54.9, 42.1, 40.8, 36.9, 30.0, 30.0, 27.7, 26.5, 23.2, 21.7, 18.8; IR (thin film/NaCl) 2935 (m), 2870 (m), 2251 (w), 1707 (s), 1597 (m), 1494 (w), 1451 (m), 1379 (m), 1317 (s), 1158 (s) $\mathrm{cm}^{-1}$; HRMS (FAB) $\mathrm{m} / \mathrm{z}$ found: 390.1744 [calc'd for $\left.\mathrm{C}_{21} \mathrm{H}_{28} \mathrm{NO}_{4} \mathrm{~S}(\mathrm{M}+\mathrm{H}): 390.1739\right]$.

\section{Preparation of alcohols 18 and 30}

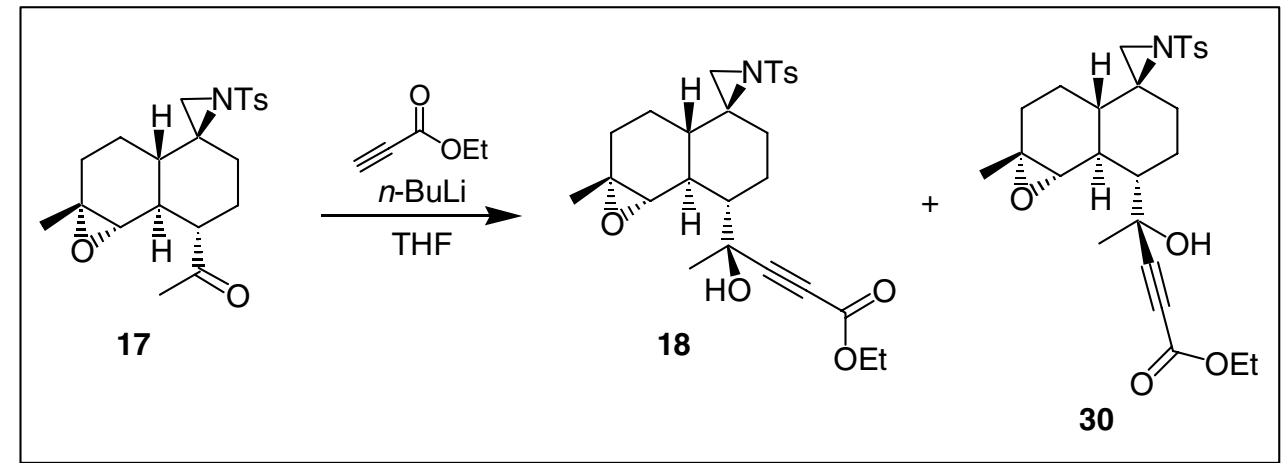

Alcohols 18 and 30. A solution of ethyl propiolate $(118 \mu \mathrm{L}, 1.16 \mathrm{mmol}, 4.0$ equiv) in THF $(10 \mathrm{~mL})$ at $-78^{\circ} \mathrm{C}$ was treated with $n$-BuLi $(2.3 \mathrm{M}$ in hexanes, $0.50 \mathrm{~mL}$, $1.16 \mathrm{mmol}, 4.0$ equiv). After $5 \mathrm{~min}, 17$ (113 mg, $0.29 \mathrm{mmol}, 1.0$ equiv) in THF (1 mL) was added. The temperature was maintained at $-78^{\circ} \mathrm{C}$ for 30 min before slowly warming to $0^{\circ} \mathrm{C}$ for an additional $1 \mathrm{hr}$. Water $(10 \mathrm{~mL})$ was added followed by EtOAc $(30 \mathrm{~mL})$. The layers were separated and the aqueous portion was extracted with EtOAc $(10 \mathrm{~mL})$. The combined organic fractions were washed with brine $(25 \mathrm{~mL})$ and dried with $\mathrm{Na}_{2} \mathrm{SO}_{4}$ 
before concentrating in vacuo. Crude NMR showed a $~ 98: 2$ mixture of diastereomers which could be purified by silica gel column chromatography (3:1 hexanes:EtOAc) to afford $18(125 \mathrm{mg}, 88 \%)$ as a foam and $\mathbf{3 0}(2 \mathrm{mg}, 1 \%)$ as a colorless oil.

Alcohol 18. ${ }^{1} \mathrm{H}$ NMR $\left(400 \mathrm{MHz}, \mathrm{CDCl}_{3}\right) \delta 7.79(\mathrm{~d}, J=8.0 \mathrm{~Hz}, 2 \mathrm{H}), 7.31(\mathrm{~d}$, $J=8.0 \mathrm{~Hz}, 2 \mathrm{H}), 4.24$ (q, J=7.2 Hz, 2H), 3.93 (s, 1H), 2.59 (s, 1H), 2.50 (s, 1H), 2.44-2.19 (comp m, 7H), 2.00-1.91 (comp m, 2H), 1.70-1.56 (comp m, 7H), 1.49-1.42 (m, 1H), 1.34-1.25 (comp m, 6H), 0.79-0.67 (m, 1H); $\left.{ }^{13} \mathrm{C} \mathrm{NMR} \mathrm{(125} \mathrm{MHz,} \mathrm{CDCl}_{3}\right) \delta 153.5,144.0$, 138.1, 129.6, 127.4, 90.3, 77.2, 72.0, 62.4, 60.4, 59.4, 54.4, 48.1, 43.3, 42.6, 36.2, 30.8, 30.0, 29.8, 25.2, 23.4, 21.7, 18.5, 14.2; IR (thin film/NaCl) 3463 (br m), 2932 (m), 2237

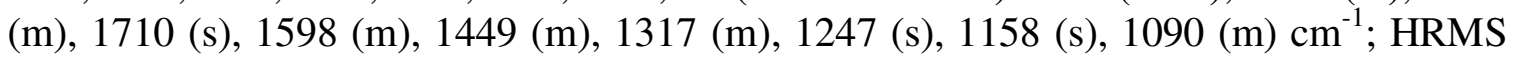
(FAB) $m / z$ found: 488.2109 [calc'd for $\mathrm{C}_{26} \mathrm{H}_{34} \mathrm{NO}_{6} \mathrm{~S}(\mathrm{M}+\mathrm{H})$ : 488.2107].

Alcohol 30. ${ }^{1} \mathrm{H}$ NMR $\left(400 \mathrm{MHz}, \mathrm{CDCl}_{3}\right) \delta 7.83(\mathrm{~d}, J=8.3 \mathrm{~Hz}, 2 \mathrm{H}), 7.30(\mathrm{~d}$, $J=8.3 \mathrm{~Hz}, 2 \mathrm{H}), 4.24(\mathrm{q}, J=7.1 \mathrm{~Hz}, 2 \mathrm{H}), 3.98$ (s, 1H), 2.51-2.26 (comp m, 8H), 2.09-1.84 (comp m, 5H), 1.65-1.51 (comp m, 4H), 1.37-1.25 (comp m,7H), 1.16 (dq, J=4.2, 12.7 $\mathrm{Hz}, 2 \mathrm{H}), 0.98-0.92(\mathrm{~m}, 1 \mathrm{H}) ;{ }^{13} \mathrm{C}$ NMR $\left(100 \mathrm{MHz}, \mathrm{CDCl}_{3}\right) \delta 153.7,144.0,137.7,129.6$, 127.7, 90.4, 77.0, 73.0, 62.3, 60.4, 58.7, 55.5, 48.2, 42.3, 41.7, 36.9, 30.3, 29.8, 29.7, 24.6, 23.4, 21.7, 19.1, 14.2; IR (thin film/NaCl) 3455 (br m), 2983 (m), 2939 (m), 2240 (m), 1710 (s), 1598 (w), 1445 (w), 1451 (m), 1370 (m), 1248 (s), 1156 (s) cm c ; HRMS (FAB) $m / z$ found: 488.2107 [calc'd for $\mathrm{C}_{26} \mathrm{H}_{34} \mathrm{NO}_{6} \mathrm{~S}(\mathrm{M}+\mathrm{H})$ : 488.2107].

\section{Preparation of ester 19}

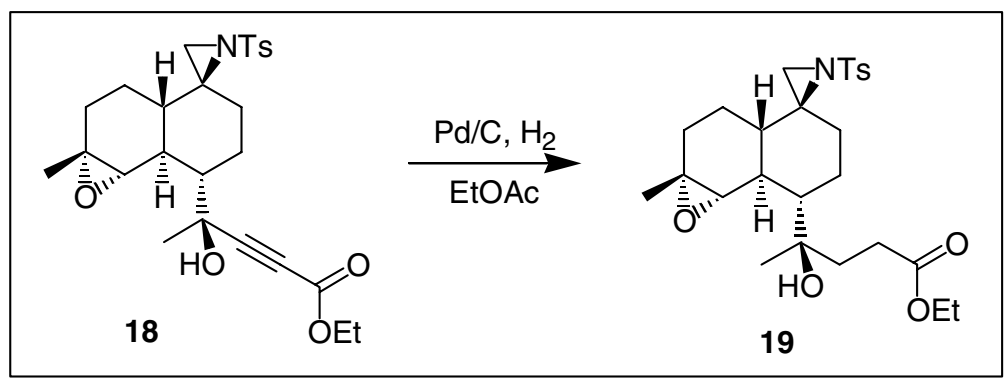

Ester 19. A solution of 18 (50 mg, $0.103 \mathrm{mmol}, 1.0$ equiv) in EtOAc (5 mL) at $0^{\circ} \mathrm{C}$ was treated with $10 \% \mathrm{Pd} / \mathrm{C}(5 \mathrm{mg})$ and an atmosphere of $\mathrm{H}_{2}$ was maintained using a balloon. After $1 \mathrm{~h}$, the mixture was filtered through celite and concentrated. The resulting colorless oil was spectroscopically pure 19 (49 mg, 98\%). ${ }^{1} \mathrm{H} \mathrm{NMR}(400 \mathrm{MHz}$, $\left.\mathrm{CDCl}_{3}\right) \delta 7.79(\mathrm{~d}, J=8.3 \mathrm{~Hz}, 2 \mathrm{H}), 7.30$ (d, $\left.J=8.6 \mathrm{~Hz}, 2 \mathrm{H}\right), 4.19-4.12$ (comp m, 3H), 2.552.18 (comp m, 9H), 1.99-1.89 (comp m, 3H), 1.81-1.54 (comp m, 6H), 1.48-1.41 (m, 1H), 1.30-1.09 (comp m, 10H), 0.79-0.68 (m, 1H); $\left.{ }^{13} \mathrm{C} \mathrm{NMR} \mathrm{(100} \mathrm{MHz,} \mathrm{CDCl}_{3}\right) \delta 175.0$, $143.9,138.2$, 129.6, 127.3 , 75.3, 60.9, 60.9, 59.3, 54.8, 47.5, 44.2, 42.9, 37.6, 36.4, 31.1, 29.8, 29.1, 28.4, 23.6, 23.3, 21.7, 18.6, 14.3; IR (thin film/NaCl) 3499 (m), $2976(\mathrm{~m})$, 2930 (m), 2875 (m), 2251 (w), 1730 (s), 1598 (w), 1451 (m), 1380 (m), 1317 (s) cm $\mathrm{cm}^{-1}$; HRMS (FAB) $m / z$ found: 492.2420 [calc'd for $\mathrm{C}_{26} \mathrm{H}_{38} \mathrm{NO}_{6} \mathrm{~S}(\mathrm{M}+\mathrm{H})$ : 492.2420]. 
Preparation of lactone 20

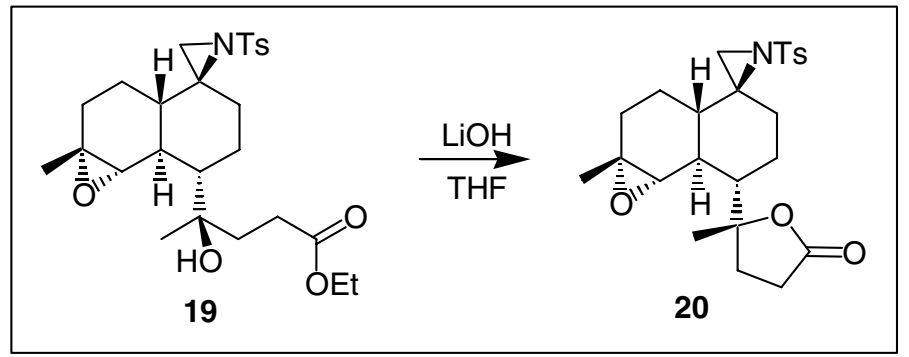

Lactone 20. A solution of $19(10 \mathrm{mg}, 0.020 \mathrm{mmol}, 1.0$ equiv) in THF (10 mL) at rt was treated with $\mathrm{LiOH}(1.5 \mathrm{mg}, 0.060 \mathrm{mmol}, 3.0$ equiv). After $12 \mathrm{hr}$, the solvent was removed in vacuo and the residue purified by silica gel column chromatography $(1: 1$ hexanes:EtOAc) to give $\mathbf{2 0}(270 \mathrm{mg}, \mathbf{9 4 \%})$ as a white solid. m.p. 200-201 ${ }^{\circ} \mathrm{C}$ (recrystallized from 1:1 hexanes:EtOAc); ${ }^{1} \mathrm{H}$ NMR $\left(500 \mathrm{MHz}, \mathrm{CDCl}_{3}\right) \delta 7.79(\mathrm{~d}, J=8.1$ $\mathrm{Hz}, 2 \mathrm{H}), 7.30(\mathrm{~d}, J=8.2 \mathrm{~Hz}, 2 \mathrm{H}), 3.70(\mathrm{~s}, 1 \mathrm{H}), 2.66-2.56(\mathrm{~m}, 1 \mathrm{H}), 2.52-2.40$ (comp m, 5H), 2.37-2.21 (comp m, 3H), 2.15-2.00 (comp m, 3H), 1.97-1.82 (comp m, 2H), 1.691.56 (comp m, 3H), 1.48-1.37 (comp m, 4H), 1.32-1.14 (comp m, 4H), 0.79-0.69 (m, $1 \mathrm{H}) ;{ }^{13} \mathrm{C}$ NMR $\left(125 \mathrm{MHz}, \mathrm{CDCl}_{3}\right) \delta 176.1,144.0,138.0,129.6,127.3,89.2,59.7,59.3$, 54.4, 49.3, 43.7, 42.4, 26.3, 35.2, 30.9, 29.8, 28.2, 27.9, 23.4, 21.7, 19.6, 18.4; IR (thin film/NaCl) 2959 (m), 2876 (m), 2252 (w), 1770 (s), 1598 (w), 1451 (m), 1387 (m), 1317 (m), $1209(\mathrm{~m}), 1158(\mathrm{~s}) \mathrm{cm}^{-1}$; HRMS (FAB) $\mathrm{m} / \mathrm{z}$ found: 446.2000 [calc'd for $\mathrm{C}_{24} \mathrm{H}_{32} \mathrm{NO}_{5} \mathrm{~S}$ $(\mathrm{M}+\mathrm{H}): 446.2001]$.

\section{Preparation of lactol 21}

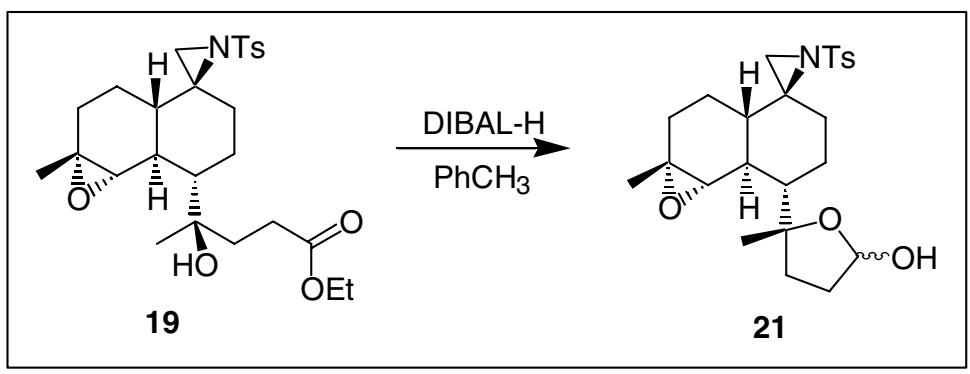

Lactol 21. To a solution of 19 (50 $\mathrm{mg}, 0.102 \mathrm{mmol}, 1.0$ equiv) in toluene (5 mL) at $0^{\circ} \mathrm{C}$ was added DIBAL-H $\left(0.5 \mathrm{M}\right.$ in $\mathrm{CH}_{2} \mathrm{Cl}_{2}, 155 \mu \mathrm{L}, 0.122 \mathrm{mmol}, 1.2$ equiv $)$. After 10 min, the solution was warmed to $\mathrm{rt}$ and stirred for an additional $2 \mathrm{~h}$. The reaction was quenched with $20 \%$ aqueous Rochelle's salt $(10 \mathrm{~mL})$ and diluted with $(25 \mathrm{~mL})$. The organic layer was washed with water $(25 \mathrm{~mL})$, brine $(25 \mathrm{~mL})$ and dried with $\mathrm{Na}_{2} \mathrm{SO}_{4}$. After concentration, the resulting residue was purified by silica gel column chromatography (3:1 then 1:1 hexanes:EtOAc) to afford 21 (35 $\mathrm{mg}, 76 \%$ ) as an amorphous, white solid. (2:1 mixture of lactol epimers) ${ }^{1} \mathrm{H} \mathrm{NMR}\left(500 \mathrm{MHz}, \mathrm{CDCl}_{3}\right) \delta$ $7.80(\mathrm{~d}, J=7.9 \mathrm{~Hz}, 2 \mathrm{H}), 7.30(\mathrm{~d}, J=7.9 \mathrm{~Hz}, 2 \mathrm{H}), 5.58-5.54$ (comp m, 0.3H), 5.52-5.48 (comp m, 0.7H), $4.05(\mathrm{~s}, 0.7 \mathrm{H}), 4.03(\mathrm{~s}, 0.3 \mathrm{H}), 2.72-2.70(\mathrm{~m}, 0.3 \mathrm{H}), 2.50-2.42$ (comp m, 4.7H), 2.31-2.18 (comp m, 3H), 2.10-1.86 (comp m, 4H), 1.80-1.69 (comp m, 2H), 1.641.56 (comp m, 4H), 1.47-1.41 (m, 1H), 1.36 (s, 1H), 1.31-1.13 (comp m, 6H), 0.80-0.69 $(\mathrm{m}, 1 \mathrm{H}) ;{ }^{13} \mathrm{C}$ NMR $\left(125 \mathrm{MHz}, \mathrm{CDCl}_{3}\right) \delta 143.9,138.3,129.6,127.4,99.5,98.4,88.3$, 87.4, 60.7, 60.6, 59.3, 59.2, 55.0, 55.0, 51.6, 50.2, 44.1, 43.9, 42.7, 42.6, 38.5, 36.5, 36.3, $36.0,32.8,32.3,31.2$, 31.2, 30.1, 30.0, 30.0, 29.6, 23.6, 23.6, 21.7, 21.5, 20.8, 18.6, 18.5; 
IR (thin film/NaCl) 3435 (m), 2929 (m), 2871 (m), 2250 (w), 1738 (m), 1599 (w), 1453 (m), $1380(\mathrm{~m}), 1318(\mathrm{~m}), 1159(\mathrm{~m}) \mathrm{cm}^{-1}$; HRMS (FAB) $\mathrm{m} / z$ found: 448.2157 [calc'd for $\left.\mathrm{C}_{24} \mathrm{H}_{34} \mathrm{NO}_{5} \mathrm{~S}(\mathrm{M}+\mathrm{H}): 448.2158\right]$.

\section{Preparation of olefin 22}

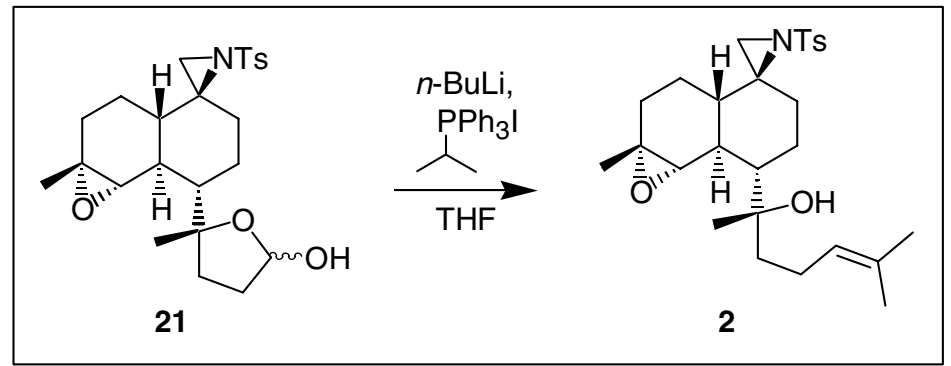

Olefin 2. A mixture of isopropyltriphenylphosponium iodide $(116 \mathrm{mg}, 0.268$ mmol, 4.0 equiv) in THF $(5 \mathrm{~mL})$ at $0^{\circ} \mathrm{C}$ was treated with $\mathrm{n}-\mathrm{BuLi}(2.3 \mathrm{M}$ in hexanes, 117 $\mu \mathrm{L}, 0.268 \mathrm{mmol}, 4.0$ equiv). After $5 \mathrm{~min}, 21$ (30 mg, $0.067 \mathrm{mmol}, 1.0$ equiv) was added as a solution in THF ( $1 \mathrm{~mL})$. The red solution was warmed to $\mathrm{rt}$ and stirred for an additional $3 \mathrm{~h}$ before quenching with water $(5 \mathrm{~mL})$ and diluting with EtOAc $(25 \mathrm{~mL})$. The aqueous layer was extracted with EtOAc $(10 \mathrm{~mL})$ and the combined organic fractions were washed with brine $(25 \mathrm{~mL})$ and dried with $\mathrm{Na}_{2} \mathrm{SO}_{4}$. After the solvent was removed in vacuo, the resulting residue was purified by silica gel column chromatography (3:1 hexanes:EtOAc) to afford $2(26 \mathrm{mg}, 81 \%)$ as a colorless oil. ${ }^{1} \mathrm{H}$ NMR (400 MHz, $\left.\mathrm{CDCl}_{3}\right) \delta 7.79(\mathrm{~d}, J=8.5 \mathrm{~Hz}, 2 \mathrm{H}), 7.30(\mathrm{~d}, J=8.1 \mathrm{~Hz}, 2 \mathrm{H}), 5.12$ (br t, $J=7.0 \mathrm{~Hz}, 1 \mathrm{H}), 4.21(\mathrm{~s}, 1 \mathrm{H}), 2.49$ (s, 1H), 2.43 (s, 3H), 2.30-1.89 (comp m, 7H), 1.721.53 (comp m, 10H), 1.47-1.36 (comp m, 3H), 1.27 (s, 3H), 1.26 (s, 3H), 1.21-1.08 (m, $1 \mathrm{H}), 0.80-0.68(\mathrm{~m}, 1 \mathrm{H}) ;{ }^{13} \mathrm{C} \mathrm{NMR}\left(125 \mathrm{MHz}, \mathrm{CDCl}_{3}\right) \delta 143.9,138.4,132.5,129.6$, 127.4, 124.1, 76.2, 61.0, 59.3, 55.1, 47.4, 44.3, 43.4, 43.0, 36.5, 31.3, 29.9, 29.4, 25.9, 23.7, 23.6, 21.7, 21.6, 18.6, 17.9; IR (thin film/NaCl) 3511 (br m), 2965 (s), 2925 (s), 2246 (w), 1598 (m), 1449 (m), 1379 (m), 1318 (s), 1208 (m), 1158 (s) cm c $^{-1}$ HRMS (FAB) $m / z$ found: 474.2679 [calc'd for $\mathrm{C}_{27} \mathrm{H}_{40} \mathrm{NO}_{4} \mathrm{~S}(\mathrm{M}+\mathrm{H})$ : 474.2678].

\section{Preparation of tetrahydrofurans 22 and 31}

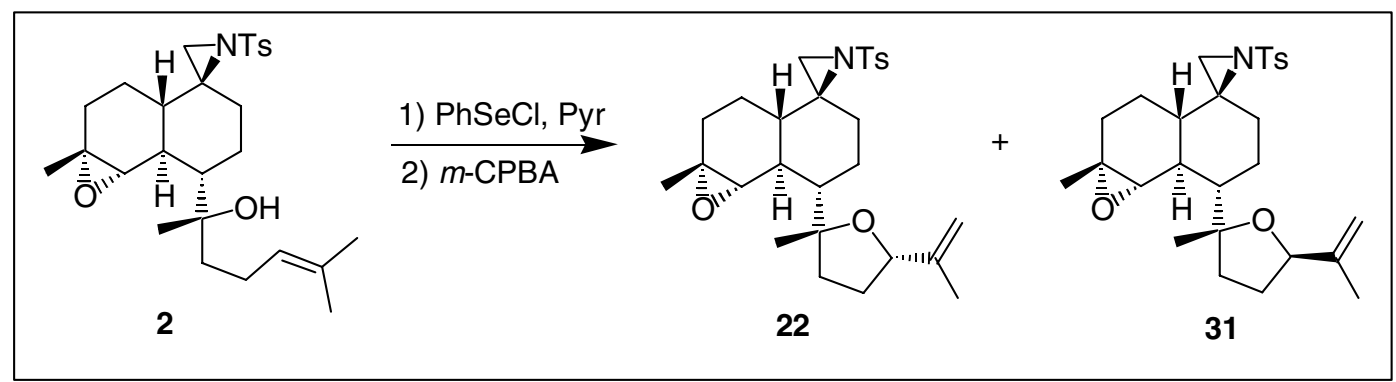

Tetrahydrofurans 22 and 31. A solution of $2(30 \mathrm{mg}, 0.063 \mathrm{mmol}, 1.0$ equiv) in $\mathrm{CH}_{2} \mathrm{Cl}_{2}(5 \mathrm{~mL})$ at $-78^{\circ} \mathrm{C}$ was treated with pyridine $(8 \mu \mathrm{L}, 0.076 \mathrm{mmol}, 1.2$ equiv $)$ and $\mathrm{PhSeCl}$ (17 mg, $0.088 \mathrm{mmol}, 1.4$ equiv). After ca. 10 minutes, saturated aqueous $\mathrm{NaHCO}_{3}(1 \mathrm{~mL})$ was added and the mixture was allowed to warm to rt. The organic layer was washed with brine $(10 \mathrm{~mL})$ and dried with $\mathrm{Na}_{2} \mathrm{SO}_{4}$. After concentration, the resulting residue was dissolved in $\mathrm{CH}_{2} \mathrm{Cl}_{2}(5 \mathrm{~mL})$, cooled to $-78^{\circ} \mathrm{C}$, and reacted with $\mathrm{m}$ - 
CPBA (15 mg, $0.088 \mathrm{mmol}, 1.4$ equiv). After ca. 20 minutes, saturated aqueous $\mathrm{NaHCO}_{3}(1 \mathrm{~mL})$ was added and the mixture was allowed to warm to rt. The organic layer was washed with brine $(10 \mathrm{~mL})$, dried with $\mathrm{Na}_{2} \mathrm{SO}_{4}$, and concentrated in vacuo. NMR analysis of the residue showed a 3:2 mixture of tetrahydrofurans which could be separated by silica gel column chromatography (9:1 then 3:1 hexanes:EtOAc) to afford 22 (major diastereomer, 48\%, $14 \mathrm{mg}$ ) and $\mathbf{3 1}(30 \%, 9 \mathrm{mg}$ ) as colorless oils.

Tetrahydrofuran 22. ${ }^{1} \mathrm{H} \mathrm{NMR}\left(400 \mathrm{MHz}, \mathrm{CDCl}_{3}\right) \delta 7.80(\mathrm{~d}, J=8.2 \mathrm{~Hz}, 2 \mathrm{H})$, $7.30(\mathrm{~d}, J=8.2 \mathrm{~Hz}, 2 \mathrm{H}), 4.91(\mathrm{~s}, 1 \mathrm{H}), 4.73(\mathrm{~s}, 1 \mathrm{H}), 4.46-4.42(\mathrm{~m}, 1 \mathrm{H}), 4.20(\mathrm{~s}, 1 \mathrm{H}), 2.50$ (s, 1H), $2.43(\mathrm{~s}, 3 \mathrm{H}), 2.29-2.23$ (comp m, 2H), $2.21(\mathrm{~s}, 1 \mathrm{H}), 2.14-2.03(\mathrm{~m}, 1 \mathrm{H}), 2.00-1.90$ (comp m, 2H), 1.75-1.53 (comp m, 9H), 1.47-1.40 (m, 1H), 1.32-1.16 (comp m, 8H), 0.81-0.68 (m, 1H); ${ }^{13} \mathrm{C}$ NMR $\left(125 \mathrm{MHz}, \mathrm{CDCl}_{3}\right) \delta 146.7,143.8,138.3,129.6,127.4$, 109.3, 86.6, 80.7, 60.8, 59.1, 55.1, 50.4, 43.9, 43.0, 38.1, 36.5, 31.3, 30.3, 30.1, 28.7, 23.4, 21.7, 19.4, 18.9, 18.5; IR (thin film/NaCl) 2965 (m), 2878 (m), 2249 (w), 1651 (w), 1599 (m), 1495 (w), 1451 (m), 1379 (m), 1321 (s), 1159 (s) cm cm $^{-1}$ HRMS (FAB) $\mathrm{m} / z$ found: 472.2522 [calc'd for $\mathrm{C}_{27} \mathrm{H}_{38} \mathrm{NO}_{4} \mathrm{~S}(\mathrm{M}+\mathrm{H})$ : 472.2522].

Tetrahydrofuran 31. ${ }^{1} \mathrm{H} \mathrm{NMR}\left(400 \mathrm{MHz}, \mathrm{CDCl}_{3}\right) \delta 7.81(\mathrm{~d}, J=8.3 \mathrm{~Hz}, 2 \mathrm{H})$, $7.31(\mathrm{~d}, J=8.3 \mathrm{~Hz}, 2 \mathrm{H}), 4.98(\mathrm{~s}, 1 \mathrm{H}), 4.74(\mathrm{~s}, 1 \mathrm{H}), 4.34(\mathrm{dd}, J=5.6,9.6 \mathrm{~Hz}, 1 \mathrm{H}), 4.14$ (s, 1H), 2.50 (s, 1H), 2.43 (s, 3H), 2.31-2.25 (comp m, 2H), 2.23 (s, 1H), 2.04-1.89 (comp m, 3H), 1.86-1.80 (comp m, 2H), 1.76-1.54 (comp m, 8H), 1.46-1.39 (m, 1H), 1.29-1.18 (comp m, 7H), 0.82-0.70 (m, 1H); ${ }^{13} \mathrm{C} \mathrm{NMR}\left(100 \mathrm{MHz}, \mathrm{CDCl}_{3}\right) \delta 146.4,143.9,138.3$, 129.6, 127.4, 109.2, 86.1, 82.5, 60.7, 59.3, 55.3, 50.8, 44.0, 42.9, 39.9, 36.6, 31.4, 30.5, 29.9, 29.9, 23.6, 21.8, 21.8, 18.8, 18.4; IR (thin film/NaCl) 2967 (m), 2931 (m), 2872 (m), 2248 (w), 1652 (w), 1598 (w), 1495 (w), 1450 (m), 1320 (m), 1159 (m) cm c $^{-1}$ HRMS (FAB) $m / z$ found: 472.2522 [calc'd for $\mathrm{C}_{27} \mathrm{H}_{38} \mathrm{NO}_{4} \mathrm{~S}(\mathrm{M}+\mathrm{H})$ : 472.2522].

\section{Preparation of amine 32}

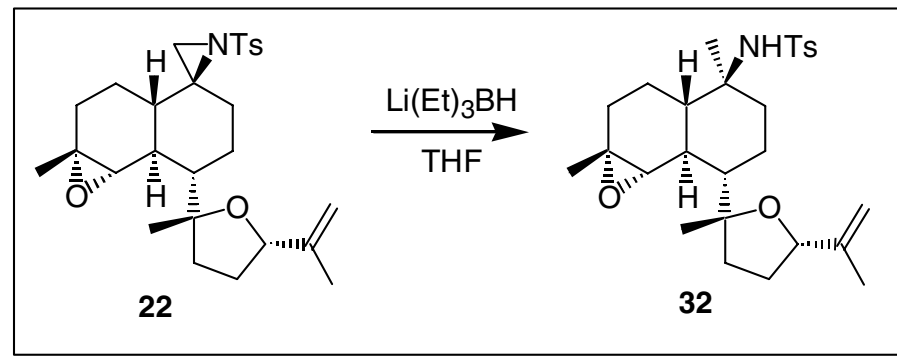

Amine 32. To a solution of aziridine $22(30 \mathrm{mg}, 0.064 \mathrm{mmol}, 1.0$ equiv $)$ in THF $(5 \mathrm{~mL})$ at $0^{\circ} \mathrm{C}$ was added a $1 \mathrm{M}$ solution of $\mathrm{Li}(\mathrm{Et})_{3} \mathrm{BH}$ in $\mathrm{THF}(191 \mu \mathrm{L}, 0.191 \mathrm{mmol}, 3.0$ equiv). The reaction mixture was stirred at $0^{\circ} \mathrm{C}$ for $1 \mathrm{~h}$ before warming to $\mathrm{rt}$ for an additional $2 \mathrm{~h}$. The reaction was quenched with $\mathrm{H}_{2} \mathrm{O}(5 \mathrm{~mL})$ and diluted with EtOAc (25 $\mathrm{mL})$. The organic layer was separated, washed with brine $(2 \times 10 \mathrm{~mL})$, and dried with $\mathrm{Na}_{2} \mathrm{SO}_{4}$. After removing the solvent in vacuo, the resulting residue was purified by silica gel column chromatography (3:1 hexanes:EtOAc) to afford 32 (26 mg, 86\% yield) as a white foam. ${ }^{1} \mathrm{H}$ NMR $\left(400 \mathrm{MHz}, \mathrm{CDCl}_{3}\right) \delta 7.77(\mathrm{~d}, J=8.1 \mathrm{~Hz}, 2 \mathrm{H}), 7.27(\mathrm{~d}, J=7.9 \mathrm{~Hz}$, 2H), $4.88(\mathrm{~s}, 1 \mathrm{H}), 4.82(\mathrm{~s}, 1 \mathrm{H}), 4.71(\mathrm{~s}, 1 \mathrm{H}), 4.43-4.38(\mathrm{~m}, 1 \mathrm{H}), 4.11(\mathrm{~s}, 1 \mathrm{H}), 2.42(\mathrm{~s}, 3 \mathrm{H})$, 2.10-1.90 (comp m, 2H), 1.76-1.42 (comp m, 12H), 1.30-1.19 (comp m, 5H), 1.13 (s, 3H), 1.12-0.96 (comp m, 5H); $\left.{ }^{13} \mathrm{C} \mathrm{NMR} \mathrm{(100} \mathrm{MHz,} \mathrm{CDCl}_{3}\right) \delta 146.7,143.0,140.9,129.7$, 127.0, 109.2, 86.7, 80.5, 61.5, 60.3, 58.8, 50.1, 47.2, 40.4, 39.2, 37.9, 30.7, 28.7, 26.7, 23.4, 21.7, 19.9, 19.3, 18.8, 18.7; IR (thin film/NaCl) 3267 (m), 2965 (m), 2871 (m), 


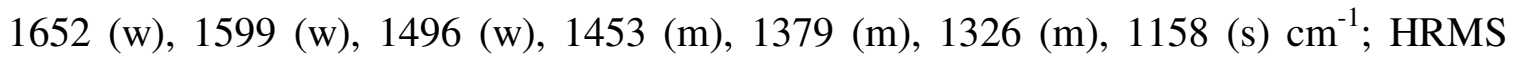
(FAB) $m / z$ found: 474.2678 [calc'd for $\mathrm{C}_{27} \mathrm{H}_{40} \mathrm{NO}_{4} \mathrm{~S}(\mathrm{M}+\mathrm{H}): 474.2678$ ].

\section{Preparation of azide 23}

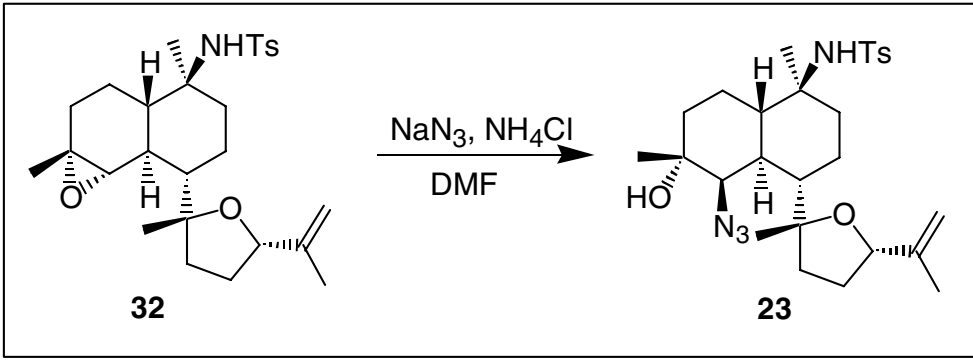

Azide 23. To a solution of 32 (30 $\mathrm{mg}, 0.063 \mathrm{mmol}, 1.0$ equiv) in DMF (7 mL) was added $\mathrm{NaN}_{3}\left(25 \mathrm{mg}, 0.378 \mathrm{mmol}, 6.0\right.$ equiv) and $\mathrm{NH}_{4} \mathrm{Cl}(10 \mathrm{mg}, 0.189 \mathrm{mmol}, 3.0$ equiv). The mixture was heated to $80^{\circ} \mathrm{C}$. After ca. $72 \mathrm{~h}$, the solution was cooled to $\mathrm{rt}$ and diluted with EtOAc $(25 \mathrm{~mL})$. After washing with water $(25 \mathrm{~mL})$ and brine $(2 \mathrm{x} 25 \mathrm{~mL})$, the organic fraction was dried with $\mathrm{Na}_{2} \mathrm{SO}_{4}$ and concentrated under reduced pressure. Purification by silica gel column chromatography (3:1 hexanes:EtOAc) afforded azide 23 (27 mg, 82\% yield) as an amorphous, white solid. ${ }^{1} \mathrm{H} \mathrm{NMR}\left(500 \mathrm{MHz}, \mathrm{CDCl}_{3}\right) \delta 7.76(\mathrm{~d}$, $J=8.4 \mathrm{~Hz}, 2 \mathrm{H}), 7.27(\mathrm{~d}, J=8.4 \mathrm{~Hz}, 2 \mathrm{H}), 4.93-4.91(\mathrm{~m}, 1 \mathrm{H}), 4.74-4.71(\mathrm{~m}, 1 \mathrm{H}), 4.46$ (dd, $J=3.4,9.5 \mathrm{~Hz}, 1 \mathrm{H}), 4.40(\mathrm{~s}, 1 \mathrm{H}), 4.07(\mathrm{~s}, 1 \mathrm{H}), 2.42$ (s, 3H), 2.11-2.05 (m, 1H), 1.95 (dd, $J=2.7,11.0 \mathrm{~Hz}, 1 \mathrm{H}), 1.85$ (ddd, $J=3.3,3.3,12.9 \mathrm{~Hz}, 1 \mathrm{H}), 1.72-1.50$ (comp m, 11H), 1.44-1.36 (comp m, 2H), 1.32-1.22 (comp m, 4H), 1.19-1.01 (comp m, 8H); ${ }^{13} \mathrm{C} \mathrm{NMR}$ $\left(125 \mathrm{MHz}_{\mathrm{CDCl}}\right) \delta 146.8,143.0,140.8,129.6,127.2,111.0,86.7,81.6,73.4,69.1$, $60.0,46.8,42.2,39.5,38.5,38.0,33.2,29.0,28.8,25.5,21.6,21.5,19.1,18.3,17.4$; IR (thin film/NaCl) $3530(\mathrm{~m}), 3271$ (m), 2966 (w), 2871 (w), 2103 (s), 1455 (w), 1383 (m), $1318(\mathrm{~m}), 1286(\mathrm{~m}), 1265(\mathrm{~m}) \mathrm{cm}^{-1}$; HRMS (FAB) $\mathrm{m} / \mathrm{z}$ found: 517.2849 [calc'd for $\left.\mathrm{C}_{27} \mathrm{H}_{41} \mathrm{~N}_{4} \mathrm{O}_{4} \mathrm{~S}(\mathrm{M}+\mathrm{H}): 517.2849\right]$.

\section{Preparation of $( \pm)$-kalihinol C (1)}

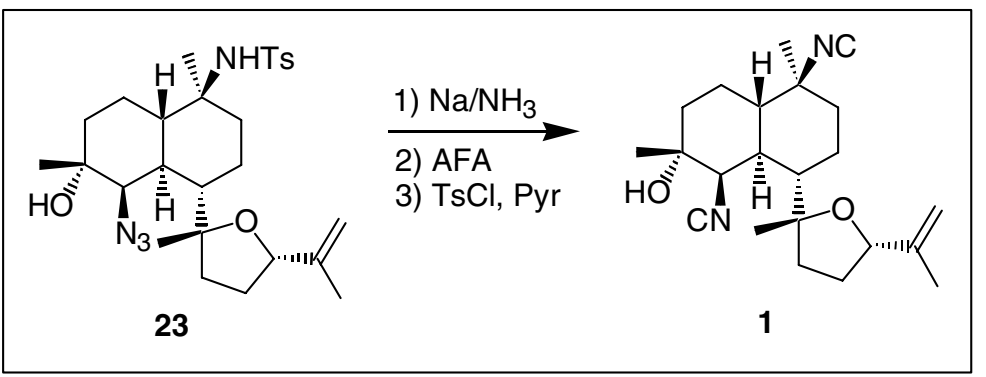

( \pm )-Kalihinol $\mathbf{C}(\mathbf{1})$. To condensed ammonia $(20 \mathrm{~mL})$ at $-78^{\circ} \mathrm{C}$ was added sodium ( $3 \mathrm{mg}, 0.116 \mathrm{mmol}, 4$ equiv). After $5 \mathrm{~min}$, the sodium/ammonia solution was added via cannula to a solution of $\mathbf{2 3}$ (15 mg, $0.029 \mathrm{mmol}, 1.0$ equiv) in THF (2 mL) and ammonia $(5 \mathrm{~mL})$ at $-78^{\circ} \mathrm{C}$. After ca. $1 \mathrm{~h}$, the reaction was quenched with solid ammonium chloride and the ammonia allowed to evaporate. The resulting residue was taken up in EtOAc $(30 \mathrm{~mL})$, washed with $0.1 \mathrm{M} \mathrm{NaOH}(2 \times 10 \mathrm{~mL})$, brine $(10 \mathrm{~mL})$ and dried with $\mathrm{Na}_{2} \mathrm{SO}_{4}$. After concentration under reduced pressure, the resulting oil was dissolved in THF (10 mL) and acetic formic anhydride (ca. $0.1 \mathrm{ml})$ was added at $\mathrm{rt}$. After 
$6 \mathrm{~h}$ the solution was concentrated under reduced pressure and the resulting residue was taken up in $\mathrm{CH}_{2} \mathrm{Cl}_{2}(5 \mathrm{~mL})$. The solution was treated with pyridine $(9 \mu \mathrm{L}, 0.116 \mathrm{mmol}$, 4.0 equiv) and $p$-toluenesulfonyl chloride ( $22 \mathrm{mg}, 0.116 \mathrm{mmol}, 4.0$ equiv) at rt. After the reaction was complete (ca. $16 \mathrm{hr}$ ), the solvent was removed in vacuo and the resulting residue was purified by silica gel column chromatography (9:1 then 3:1 hexanes:EtOAc) to afford 1 (4 mg, 39\% yield from 23 ) as a colorless oil. ${ }^{1} \mathrm{H}$ NMR $\left(500 \mathrm{MHz}, \mathrm{CDCl}_{3}\right.$, referenced to 77.0) $\delta 5.04(\mathrm{~s}, 1 \mathrm{H}), 4.77(\mathrm{~s}, 1 \mathrm{H}), 4.50(\mathrm{br} \mathrm{s}, 1 \mathrm{H}), 4.41(\mathrm{dd}, J=3.6,9.4 \mathrm{~Hz}$, $1 \mathrm{H}), 1.73(\mathrm{~s}, 3 \mathrm{H}), 1.43(\mathrm{~s}, 3 \mathrm{H}), 1.34$ (br s, 3H), 1.05 (s, 3H), 2.13-1.04 (complex); ${ }^{13} \mathrm{C}$ NMR $\left(125 \mathrm{MHz}, \mathrm{CDCl}_{3}\right) \delta 146.27,109.98,86.23,80.85,70.59,63.18(\mathrm{t}, J=5 \mathrm{~Hz}), 59.91$ (t, $J=5 \mathrm{~Hz}), 46.31,42.18,39.93,38.18,36.08,32.65,28.82$, 28.74, 24.30, 21.65, 20.73, 18.25, 18.16; IR (thin film/NaCl) 3416 (br m), 2968 (m), 2875 (m), 2137 (m), 1653 (w), 1455 (m), $1384(\mathrm{~m}), 1273$ (w), 1189 (w), $1101(\mathrm{w}) \mathrm{cm}^{-1}$; HRMS (FAB) $\mathrm{m} / \mathrm{z}$ found: 330.2433 [calc'd for $\mathrm{C}_{21} \mathrm{H}_{32} \mathrm{NO}_{2}(\mathrm{M}-\mathrm{NC})^{+}: 330.2433$ ]. 